\title{
Coupling of the phase field approach to the Armstrong-Frederick model for the simulation of ductile damage under cyclic load
}

\author{
Serhat Aygün ${ }^{1}$, Tillmann Wiegold ${ }^{2}$ and Sandra Klinge ${ }^{1, *}$ \\ ${ }^{1}$ Chair of Structural Mechanics and Analysis, TU Berlin, \\ Straße des 17. Juni 135, 10623 Berlin, Germany \\ ${ }^{2}$ Institute of Mechanics, TU Dortmund University, \\ Leonhard-Euler-Straße 5, 44227 Dortmund, Germany
}

June 7, 2021

\begin{abstract}
The present contribution proposes a thermodynamically consistent model for the simulation of the ductile damage. The model couples the phase field method of fracture to the Armstrong-Frederick plasticity model with kinematic hardening. The latter is particularly suitable for simulating the material behavior under a cyclic load. The model relies on the minimum principle of the dissipation potential. However, the application of this approach is challenging since potentials of coupled methods are defined in different spaces: The dissipation potential of the phase field model is expressed in terms of rates of internal variables, whereas the Armstrong-Frederick model proposes a formulation depending on thermodynamic forces. For this reason, a unique formulation requires the Legendre transformation of one of the potentials. The present work performs the transformation of the Armstrong-Frederick potential, such that final formulation is only expressed in the space of rates of internal variables. With the assumption for the free energy and the joint dissipation potential at hand, the derivation of evolution equations is straightforward. The application of the model is illustrated by selected numerical examples studying the material response for different load constellations and sample geometries. The paper provides a comparison with the experimental results as well.
\end{abstract}

Keywords: crack mechanics; cyclic loading; ductility; elastic-plastic material; fracture

${ }^{*}$ Correspondence: sandra.klinge@tu-berlin.de 


\section{Introduction}

Fatigue failure significantly influences the safety measures during the service life of engineering structures. It particularly holds for structures subjected to alternate loading with high amplitudes, which is an often situation when speaking of transportation vehicles. Dependent on the number of cycles and load amplitude, the interplay of the brittle and ductile failure takes place. However, the present contribution focuses on the low cycle fatigue where the role of plasticity is essential. The high complexity of the ductile damage under the cyclic load strongly motivates the virtual testing of materials on the basis of the computer simulations. The present work envisages a coupling of the phase field method of fracture to the Armstrong-Frederick model of plasticity with the kinematic hardening to this end.

The base for the phase field model of fracture has been set in the work by Francfort and Marigo (1998) proposing the formulation of brittle fracture based solely on Griffith's idea of competition between elastic and fracture energy. This work initiated a large group of further contributions related to the numerical implementation of the concept proposed (Bourdin and Chambolle, 2000, Negri and Paolini, 2001; Fraternali, 2007, Schmidt et al., 2009). Amongst others, Ambrosio-Tortorelli regularizations have become ubiquitous (Ambrosio and Tortorelli, 1990, 1992, Bourdin et al., 2000). These approaches are nowadays known as phase-field models of fracture and share several common features with the approaches resulting from Ginzburg-Landau models for phase transition (Karma et al. 2001). These regularizations have been applied to a wide variety of fracture problems including fracture of thermal and drying cracks (Maurini et al. 2013: Bourdin et al., 2014), ferro-magnetic and piezo-electric materials (Abdollahi and Arias, 2012||Wilson et al. 2013) and hydraulic fracturing (Bourdin et al. 2012 |Wheeler et al., 2014: Wilson and Landis, 2016). These models also been enhanced to account for cohesive effects (Crismale and Lazzaroni, 2016; Freddi and Iurlano, 2017), ductile behavior (Alessi et al., 2014; Miehe et al., 2015 Ambati et al., 2015a), large deformations (Ambati et al. 2015b: Miehe et al. 2016 Borden et al. | 2016), quasi-brittle damage (Narayan and Anand, 2019) and anisotropy (Li et al., 2014).

Some of previous works have also considered coupling of plasticity to the phase field method (Alessi et al., 2014, Ambati et al., 2015a b; Borden et al. 2016 Miehe et al., 2016, 2015). However, the current contribution will focus more precisely on the plasticity under the cyclic load where the ArmstrongFrederick model is a widely accepted concept (Bari and Hassan, 2001, Khan and Jackson, 1999; Kobayashi and Ohno, 2002, Puzrin and Houlsby, 2001). Some of the works in this field deal with so-called generalized representations, where either the classical Armstrong-Frederick model is extended to include several Armstrong-Frederick terms (Bari and Hassan, 2001), or where the application of multiple yield surfaces is envisaged (Puzrin and Houlsby, 2001). Another frequently used variant of the Armstrong-Frederick model has been presented by Ohno and Wang (1993) and by Kobayashi and Ohno (2002). The effects of the kinematic hardening are nowadays also investigated within the framework of large deformations (Lührs et al., 1997, Svendsen et al., 1998, Lion, 2000: Mollica et al. 2001).

The Armstrong-Frederick model coupled to the phase field method is a promising approach inheriting the advantages of both incorporated techniques. 
However, the numerical implementation of this coupled approach brings with it several challenges such as the definition of a unique framework for both setups, the derivation of coupled evolution equations, the distinction between the tension and compression mode and certainly the development of the computationally efficient algorithm. Some of these issues are discussed in the present contribution which is structured as follows. An overview on the phase field method of fracture is presented in Sect. 2, whereas Sect. 3 introduces the Armstrong-Frederick model of plasticity. Furthermore, the Armstrong-Frederick evolution equations are derived by using two versions of the minimum principle of dissipation potential (Sects. 4 and 5). The concept for the coupling of two methods is presented in Sect. 6 and complemented by explaining the scheme for the calculation of the plasticity multiplier on the basis of the consistency condition (Sect. 7). Details on the numerical implementation including the global part and the material point part are presented in Sect. 8. Eventually, the paper also includes numerical examples simulating the tests with the uniformly increasing and the cyclic load. The contribution finishes with conclusions and an outlook.

\section{Diffusive crack topology}

The derivation of the coupled method requires a short recapitulation of separate approaches, which is done in two subsequent sections. Sect. 2 provides an overview of the phase field method, whereas Sect. 3 focuses on the ArmstrongFrederick model.

The present work uses the phase field framework proposed by Miehe and coworkers (Miehe et al., 2010a b) as a basis. This approach supposes the diffusive (regularized) crack topology instead of the sharp crack topology leading to serious difficulties in the numerical implementation due to the lack of the continuity and differentiability. It relies on the evaluation of the damage variable $d$, which continuously changes in the range $[0,1]$, where zero-value corresponds to the intact material and the unity-value to the fully broken state. The approach starts by postulating an expression for the crack surface density function depending on the damage variable $d$

$$
\gamma(d, \nabla d)=\frac{1}{2 l} d^{2}+\frac{l}{2}|\nabla d|^{2}
$$

which is furthermore used to define the total crack surface $A$ for the entire body $\mathcal{B}$

$$
A(d)=\int_{\mathcal{B}} \gamma(d, \nabla d) d V
$$

An assumption that the crack propagation is a fully dissipative process yields to the conclusion that the constitutive dissipation potential has to be proportional to the rate of crack surface density

$$
\Phi=g_{\mathrm{c}} \dot{\gamma}(\dot{d}, \nabla \dot{d}, d, \nabla d),
$$

where parameter $g_{\mathrm{c}}$ is related to the critical Griffith-type fracture energy and can be seen as a constitutive threshold value. However, this potential still does not guarantee that the damage is an increasing function. For this reason, the potential is extended by introducing the penalty term 


$$
P(\dot{d})=\frac{k_{\mathrm{p}}}{2}\langle\dot{d}\rangle_{-}^{2} .
$$

Here, the negative Macaulay brackets $\langle\bullet\rangle_{-}=(\bullet-|\bullet|) / 2$ assure that the penalty term only activates for a negative damage evolution and the constant $k_{\mathrm{p}}$ has to be chosen as high as possible in order to stipulate the condition $\dot{d} \geq 0$. Along with the penalty term (4), the damage potential turns into

$$
\Phi^{\mathrm{d}}=\Phi+P(\dot{d})=g_{\mathrm{c}} \dot{\gamma}(\dot{d}, \nabla \dot{d}, d, \nabla d)+\frac{k_{\mathrm{p}}}{2}\langle\dot{d}\rangle_{-}^{2} .
$$

In a further step, the model focuses on the definition of the free energy and its reduction on the basis of function $\omega=\left((1-d)^{2}+k_{\mathrm{d}}\right)$. Here, the positive constant $k_{\mathrm{d}}$ prevents the energy to become identical to zero at a fully broken state. Moreover, the reduction function only affects the tension part of the energy which corresponds to the concept of the anisotropic degradation of energy

$$
\Psi^{\mathrm{d}}(\boldsymbol{\epsilon}, d)=\omega(d) \Psi_{+}(\boldsymbol{\epsilon})+\Psi_{-}(\boldsymbol{\epsilon}) .
$$

The additive split of the energy into a tension part $\Psi_{+}(\boldsymbol{\epsilon})$ and a compression part $\Psi_{-}(\boldsymbol{\epsilon})$, is of the special interest for simulating the cyclic behavior where the crack closure in the compression mode has to be considered. The definitions of the positive and negative energy parts of the free energy are based on the spectral decomposition of the strain tensor

$$
\boldsymbol{\epsilon}_{ \pm}=\sum_{i}\left\langle\epsilon_{i}\right\rangle_{ \pm} \boldsymbol{n}_{i} \otimes \boldsymbol{n}_{i}
$$

where $\epsilon_{i}$ are the principal strains, $\boldsymbol{n}_{i}$ are the principal strain directions and $i$ is the summation index. Equation (7) uses positive and negative Macaulay brackets. Positive ones are defined as $\langle\bullet\rangle_{+}=(\bullet+|\bullet|) / 2$, whereas the negative ones have already been used in Eq. (4). The stresses corresponding to (7) are then defined as

$$
\boldsymbol{\sigma}=\frac{\partial \Psi^{\mathrm{d}}}{\partial \boldsymbol{\epsilon}}=\omega(d) \boldsymbol{\sigma}_{+}+\boldsymbol{\sigma}_{-}
$$

such that it holds $\sigma_{ \pm}=\frac{\partial \Psi_{ \pm}}{\partial \epsilon}$. For a case of an isotropic material characterized by Lamé constants $\lambda$ and $\mu$, the energy spit turns into

$$
\Psi_{ \pm}(\boldsymbol{\epsilon})=\frac{1}{2} \lambda\left(\operatorname{tr}\langle\boldsymbol{\epsilon}\rangle_{ \pm}\right)^{2}+\mu \operatorname{tr}\left(\langle\boldsymbol{\epsilon}\rangle_{ \pm}^{2}\right)
$$

with the corresponding equilibrium equation and stresses

$$
\begin{gathered}
\operatorname{Div}\left[\omega(d) \boldsymbol{\sigma}_{+}+\boldsymbol{\sigma}_{-}\right]=\mathbf{0}, \\
\boldsymbol{\sigma}_{ \pm}=\sum_{i}\left[\lambda \operatorname{tr}\langle\boldsymbol{\epsilon}\rangle_{ \pm}+2 \mu\left\langle\epsilon_{i}\right\rangle_{ \pm}\right] \boldsymbol{n}_{i} \otimes \boldsymbol{n}_{i} .
\end{gathered}
$$

Since the free energy and the dissipation potential are known, the minimum principle of dissipation potential is used to derive the evolution equation for the internal parameter, namely 


$$
\min _{\dot{d}}\left(\mathcal{L}^{\mathrm{MDP}}=\dot{\Psi}^{\mathrm{d}}(\dot{\boldsymbol{\epsilon}}, \dot{d})+\Phi^{\mathrm{d}}(d, \dot{d})\right) \quad \Rightarrow \quad \frac{g_{c}}{l}\left[d-l^{2} \Delta d\right]+\left[\omega^{\prime} \Psi_{+}+k_{\mathrm{p}}\langle\dot{d}\rangle_{-}\right]=0 .
$$

The equilibrium equation $10 \mathrm{p}$ together with the evolution equation $12 \mathrm{~b}$ defines the strong problem of brittle fracture.

\section{The Armstrong-Frederick kinematic harden- ing model}

The Armstrong-Frederick kinematic hardening model (Armstrong and Frederick, 1966) is widely accepted to simulate the characteristic phenomena of the hardening behavior of metals, namely the Bauschinger and the ratcheting effect. Within the framework of the small strain plasticity, the formulation of the Armstrong-Frederick model starts with the typical assumption for the additive decomposition of strain tensor $\epsilon$ into an elastic part $\epsilon^{\mathrm{e}}$ and a plastic part $\epsilon^{\mathrm{p}}$, namely $\boldsymbol{\epsilon}=\boldsymbol{\epsilon}^{\mathrm{e}}+\boldsymbol{\epsilon}^{\mathrm{p}}$. However, the description of the hardening effects requires the introduction of an additional strain-like internal variable $\boldsymbol{\xi}$. According to Lion (Lion, 2000), $\xi$ relates to local viscoelastic deformations induced by dislocations. In rheological models, it is simulated as a damping element connected in series to an elastic spring. These are additionally coupled to a friction element in parallel (Dettmer and Reese, 2004). This internal variable is responsible for the change of material stiffness depending on the loading history and is incorporated in the Helmholtz free energy, which now includes two parts: the elastic energy $\Psi^{\mathrm{el}}\left(\boldsymbol{\epsilon}^{\mathrm{e}}\right)$ and free energy due to the hardening $\Psi^{\text {hard }}(\boldsymbol{\xi})$, both in the quadratic form

$$
\Psi^{\mathrm{AF}}=\Psi^{\mathrm{el}}\left(\boldsymbol{\epsilon}^{\mathrm{e}}\right)+\Psi^{\mathrm{hard}}(\boldsymbol{\xi})=\frac{1}{2}\left(\boldsymbol{\epsilon}-\boldsymbol{\epsilon}^{\mathrm{p}}\right): \mathbb{C}:\left(\boldsymbol{\epsilon}-\boldsymbol{\epsilon}^{\mathrm{p}}\right)+\frac{1}{2} c \boldsymbol{\xi}: \boldsymbol{\xi} .
$$

Here, $\mathbb{C}$ is the fourth order elasticity tensor and $c$ represents the kinematic hardening modulus. The Helmholtz free energy serves furthermore as a basis for the formulation of dissipation which, in the case of an isothermal process, only depends on the elastic power $(\boldsymbol{\sigma}: \dot{\boldsymbol{\epsilon}})$ and free energy rate $\left(\dot{\Psi}^{\mathrm{AF}}\right)$

$$
\mathcal{D}=\sigma: \dot{\boldsymbol{\epsilon}}-\dot{\Psi}^{\mathrm{AF}} \geq 0 .
$$

In the concrete case of the Armstrong-Frederick model, the dissipation turns into:

$$
\left(\boldsymbol{\sigma}-\frac{\partial \Psi^{\mathrm{AF}}}{\partial \boldsymbol{\epsilon}}\right): \dot{\boldsymbol{\epsilon}}-\frac{\partial \Psi^{\mathrm{AF}}}{\partial \boldsymbol{\epsilon}^{\mathrm{p}}}: \dot{\boldsymbol{\epsilon}}^{\mathrm{p}}-\frac{\partial \Psi^{\mathrm{AF}}}{\partial \boldsymbol{\xi}}: \dot{\boldsymbol{\xi}} \geq 0,
$$

which yields two groups of consequences. The first consequence is the constitutive law $\sigma=\frac{\partial \Psi^{\mathrm{AF}}}{\partial \epsilon}$, whereas the second consequence defines the driving forces of internal variables:

$$
\begin{gathered}
\boldsymbol{q}_{\boldsymbol{\epsilon}^{\mathrm{P}}}=-\frac{\partial \Psi^{\mathrm{AF}}}{\partial \boldsymbol{\epsilon}^{\mathrm{P}}}=\mathbb{C}:\left[\boldsymbol{\epsilon}-\boldsymbol{\epsilon}^{\mathrm{P}}\right]=\boldsymbol{\sigma}, \\
\boldsymbol{q}_{\boldsymbol{\xi}}=-\frac{\partial \Psi^{\mathrm{AF}}}{\partial \boldsymbol{\xi}}=-c \boldsymbol{\xi}=\boldsymbol{\chi} .
\end{gathered}
$$


The later definition corresponds to the back stress and gives insight into the physical meaning of material parameter $c$. It is a proportionality constant relating the back stress $\chi$ to the strain-like quantity $\xi$. In analogy to the constitutive law of elastic materials, $c$ represents the kinematic hardening modulus. A shorter notation for internal variables $\boldsymbol{\nu}=\left\{\boldsymbol{\epsilon}^{\mathrm{p}}, \boldsymbol{\xi}\right\}$ and for corresponding driving forces $\boldsymbol{q}=\{\boldsymbol{\sigma}, \boldsymbol{\chi}\}$, together with the constitutive law for stresses, yields the result for the so-called reduced dissipation

$$
\mathcal{D}^{\text {red }}=\boldsymbol{q}: \dot{\boldsymbol{\nu}}=\boldsymbol{\sigma}: \dot{\boldsymbol{\epsilon}}^{\mathrm{p}}+\chi: \dot{\boldsymbol{\xi}} \geq 0 .
$$

The Armstrong-Frederick model is eventually completed by introducing the yield locus formula

$$
\Omega^{\mathrm{AF}}=\|\overline{\boldsymbol{\sigma}}-\bar{\chi}\|-\bar{\sigma}_{\mathrm{Y}}=0, \quad \bar{\sigma}_{\mathrm{Y}}=\sqrt{2 / 3} \sigma_{\mathrm{Y}}
$$

where symbol $\|\bullet\|=\sqrt{\bullet: \bullet}$ denotes the Frobenius norm, $\overline{\boldsymbol{\sigma}}$ and $\bar{\chi}$ are deviatoric parts of stresses $\boldsymbol{\sigma}$ and back stresses $\chi$ and $\sigma_{\mathrm{Y}}$ is the yield limit. Condition 19 defines the admissible set of driving forces yielding inelastic deformations.

\section{Derivation of the evolution equations based on the minimum principle for the dissipation potential in terms of driving forces}

The completion of the Armstrong-Frederick model previously described also requires the derivation of evolution equations which can be accomplished by using different concepts. One possibility is to follow the minimum principle of dissipation potential (MDP) as proposed in works by Dettmer and Reese (2004) and by Aygün and Klinge (2020)

$$
\begin{gathered}
\min _{\boldsymbol{q}}\left(\mathcal{L}^{\mathrm{MDP}}=-\boldsymbol{q}: \dot{\boldsymbol{\nu}}+\Phi^{\mathrm{AF}^{*}}(\boldsymbol{\nu}, \boldsymbol{q})\right), \\
\Phi^{\mathrm{AF}^{*}}(\boldsymbol{\nu}, \boldsymbol{q})=a\|\overline{\boldsymbol{\sigma}}-\bar{\chi}\|+\frac{1}{2 b}\|\bar{\chi}\|^{2} .
\end{gathered}
$$

The dissipation potential (21) is inspired by the expression for the yield locus 19 but additionally includes a term depending on the norm of driving force $\chi$. Symbols $a$ and $b$ denote material parameters. However, the subsequent derivations will show that parameter $a$ does not influence the evolution equations, whereas parameter $b$ plays an important role and represents pseudo-viscoelasticty. Superscript $*$ indicates that a formulation in terms of driving forces is chosen. The Lagrangian corresponding to the minimization problem (20) and (21) has the form

$$
\mathcal{L}^{\mathrm{MDP}^{*}}=-\boldsymbol{\sigma}: \dot{\boldsymbol{\epsilon}}^{\mathrm{p}}-\chi: \dot{\boldsymbol{\xi}}+\Phi^{\mathrm{AF}^{*}}(\boldsymbol{\nu}, \boldsymbol{q}),
$$

which yields the following stationary conditions

$$
\frac{\partial \mathcal{L}^{\mathrm{MDP}}{ }^{*}}{\partial \boldsymbol{q}}=\left(\begin{array}{c}
\frac{\partial \mathcal{L}^{\mathrm{MDP}}}{\partial \boldsymbol{\sigma}} \\
\frac{\partial \mathcal{L}^{\mathrm{MDP}}}{\partial \boldsymbol{\chi}}
\end{array}\right)=\left(\begin{array}{c}
-\dot{\boldsymbol{\epsilon}}^{\mathrm{p}}+\frac{\partial \Phi^{\mathrm{AF}}}{\partial \boldsymbol{\sigma}^{*}} \\
-\dot{\boldsymbol{\xi}}+\frac{\partial \Phi^{\mathrm{AF}}}{\partial \boldsymbol{\chi}}
\end{array}\right)=\mathbf{0} .
$$


The first equation in (23) determines the evolution of plastic strains and shows that the rate $\dot{\boldsymbol{\epsilon}}^{\mathrm{p}}$ is equal to the derivative of dissipation potential with respect to corresponding driving force: $\dot{\boldsymbol{\epsilon}}^{\mathrm{p}}=\frac{\partial \Phi^{\mathrm{AF}}}{\partial \boldsymbol{\sigma}}=\frac{\overline{\boldsymbol{\sigma}}-\overline{\boldsymbol{\chi}}}{\|\overline{\boldsymbol{\sigma}}-\overline{\boldsymbol{\chi}}\|}$. However, the received derivative is not uniquely defined and only contains the information on the direction of the flow of the plastic strains, not on the magnitude. For that reason, the right-hand side expression is scaled by the plastic multiplier $\lambda$ which yields the standard solution

$$
\dot{\boldsymbol{\epsilon}}^{\mathrm{p}}=\frac{\partial \Phi^{\mathrm{AF}^{*}}}{\partial \boldsymbol{\sigma}}=\lambda \frac{\overline{\boldsymbol{\sigma}}-\overline{\boldsymbol{\chi}}}{\|\overline{\boldsymbol{\sigma}}-\overline{\boldsymbol{\chi}}\|} .
$$

The second equation in 23 determines the evolution of the internal variable $\boldsymbol{\xi}$. Here, the same argumentation as in Eq. (24) is used for the first term, whereas the second term is uniquely determined and does not need to be scaled with the multiplier $\lambda$

$$
\dot{\boldsymbol{\xi}}=\frac{\partial \Phi^{\mathrm{AF}}}{\partial \chi}=-\lambda \frac{\overline{\boldsymbol{\sigma}}-\overline{\boldsymbol{\chi}}}{\|\overline{\boldsymbol{\sigma}}-\bar{\chi}\|}+\frac{1}{b} \overline{\boldsymbol{\chi}}=-\dot{\boldsymbol{\epsilon}}^{\mathrm{p}}+\frac{1}{b} \bar{\chi} \quad \Rightarrow \quad \bar{\chi}=b\left(\dot{\boldsymbol{\xi}}+\dot{\boldsymbol{\epsilon}}^{\mathrm{p}}\right) .
$$

Equations (24) and (25) a show the deviatoric character of internal variables $\epsilon^{\mathrm{p}}$ and $\boldsymbol{\xi}$. Moreover, Eq. 25p b represents a constitutive law typical of a viscous material with the viscosity $b$. Finally, Eq. (17) along with Eq. (25) a provides the evolution equation for back stress $\chi$ which can also be identified as a deviatoric quantity:

$$
\dot{\chi}=-c \dot{\boldsymbol{\xi}}=c \dot{\boldsymbol{\epsilon}}^{\mathrm{p}}-\frac{c}{b} \overline{\boldsymbol{\chi}}=\dot{\bar{\chi}}
$$

The MDP-approach differs from the common approach treating the yield locus equation $\Omega$ as a subsidiary condition within the Lagrange formalism of the constrained optimization. The main difference manifests itself in the evolution equations taking the form $\dot{\boldsymbol{\nu}}=\lambda \frac{\partial \Omega}{\partial \boldsymbol{q}}$ with $\lambda$ as the Lagrange multiplier. An important advantage of the MDP-approach compared to the constrained optimization method is that it allows a standardized variable transformation, which is often a useful tool in coupling strategies.

\section{Derivation of the evolution equations based on the minimum principle for the dissipation potential in terms of rates of internal variables}

Both models summarized in Sects. 2 and 4 apply the minimum principle of dissipation potential. However, the phase field model uses a formulation in terms of the rate of internal variables, whereas the Armstrong-Frederick model minimizes the dissipation potential in terms of driving forces. Naturally, the coupling procedure requires a unique formulation in a single space. The present contribution deals with the approach in terms of velocities, such that the transformation of the potential $\Phi^{\mathrm{AF}^{*}}(\boldsymbol{\nu}, \boldsymbol{q})$ into the space of rates is necessary. This type of exchange is conducted on the basis of Legendre transformation (LT) defining the new potential as follows 


$$
\begin{gathered}
\Phi^{\mathrm{AF}}(\boldsymbol{\nu}, \dot{\boldsymbol{\nu}})=\max _{\boldsymbol{q}}\left\{\mathcal{L}^{\mathrm{LT}}=\boldsymbol{q}: \dot{\boldsymbol{\nu}}-\Phi^{\mathrm{AF}^{*}}(\boldsymbol{\nu}, \boldsymbol{q})\right\}, \\
\mathcal{L}^{\mathrm{LT}}=\boldsymbol{\sigma}: \dot{\boldsymbol{\epsilon}}^{\mathrm{p}}+\chi: \dot{\boldsymbol{\xi}}-a\|\overline{\boldsymbol{\sigma}}-\overline{\boldsymbol{\chi}}\|-\frac{1}{2 b}\|\overline{\boldsymbol{\chi}}\|^{2} .
\end{gathered}
$$

The maximization procedure relies on two stationary conditions, the first of which gives a relationship for rates of plastic deformations

$$
\frac{\partial \mathcal{L}^{\mathrm{LT}}}{\partial \boldsymbol{\sigma}}=\dot{\boldsymbol{\epsilon}}^{\mathrm{p}}-\lambda \frac{\overline{\boldsymbol{\sigma}}-\overline{\boldsymbol{\chi}}}{\|\overline{\boldsymbol{\sigma}}-\overline{\boldsymbol{\chi}}\|}=0 .
$$

Bearing in mind that the multiplier $\lambda$ is a scalar, Eq. $(29)$ shows that rate $\dot{\boldsymbol{\epsilon}}^{\mathrm{p}}$ and difference $\overline{\boldsymbol{\sigma}}-\bar{\chi}$ are coaxial. In other words, it holds that $\frac{\dot{\epsilon}^{\mathrm{p}}}{\left\|\dot{\epsilon}^{\mathrm{P}}\right\|}=\frac{\overline{\boldsymbol{\sigma}}-\bar{\chi}}{\|\overline{\boldsymbol{\sigma}}-\overline{\boldsymbol{\chi}}\|}$. Moreover, the plastic flow only occurs if the stress state fulfills the yield locus equation 19 such that the norm $\|\overline{\boldsymbol{\sigma}}-\overline{\boldsymbol{\chi}}\|$ can be replaced by the yield limit $\bar{\sigma}_{Y}$. Equation $29 p$ is then rewritten as follows

$$
\overline{\boldsymbol{\sigma}}-\bar{\chi}=\bar{\sigma}_{\mathrm{Y}} \frac{\dot{\boldsymbol{\epsilon}}^{\mathrm{p}}}{\left\|\dot{\boldsymbol{\epsilon}}^{\mathrm{p}}\right\|} \quad \Rightarrow \quad \overline{\boldsymbol{\sigma}}=\bar{\sigma}_{\mathrm{Y}} \frac{\dot{\boldsymbol{\epsilon}}^{\mathrm{p}}}{\left\|\dot{\boldsymbol{\epsilon}}^{\mathrm{p}}\right\|}+\bar{\chi} .
$$

On the other hand, the second stationary condition

$$
\frac{\partial \mathcal{L}^{\mathrm{LT}}}{\partial \chi}=\dot{\boldsymbol{\xi}}+\lambda \frac{\overline{\boldsymbol{\sigma}}-\overline{\boldsymbol{\chi}}}{\|\overline{\boldsymbol{\sigma}}-\bar{\chi}\|}-\frac{1}{b} \overline{\boldsymbol{\chi}}=0 \quad \Rightarrow \quad \bar{\chi}=b \dot{\boldsymbol{\xi}}+b \dot{\boldsymbol{\epsilon}}^{\mathrm{p}}
$$

together with $30 \mathrm{~b}$ leads to the final expression for deviatoric stresses

$$
\overline{\boldsymbol{\sigma}}=\bar{\sigma}_{\mathrm{Y}} \frac{\dot{\boldsymbol{\epsilon}}^{\mathrm{p}}}{\left\|\dot{\boldsymbol{\epsilon}}^{\mathrm{p}}\right\|}+b \dot{\boldsymbol{\xi}}+b \dot{\boldsymbol{\epsilon}}^{\mathrm{p}} .
$$

Driving forces $31 \mathrm{~b}$ and 32 are now inserted into 28), which reads the desired dissipation potential in terms of the internal variables and their rates

$$
\Phi^{\mathrm{AF}}(\boldsymbol{\nu}, \dot{\boldsymbol{\nu}})=\bar{\sigma}_{\mathrm{Y}}\left\|\dot{\boldsymbol{\epsilon}}^{\mathrm{p}}\right\|+\frac{b}{2}\left\|\dot{\boldsymbol{\epsilon}}^{\mathrm{p}}\right\|^{2}+\frac{b}{2}\|\dot{\boldsymbol{\xi}}\|^{2}+b \dot{\boldsymbol{\epsilon}}^{\mathrm{p}}: \dot{\boldsymbol{\xi}} .
$$

More details on derivation are presented in Appendix A Result (33) now enables the formulation of the new minimization problem

$$
\min _{\dot{\nu}}\left(\mathcal{L}^{\mathrm{MDP}}=\dot{\Psi}^{\mathrm{AF}}+\Phi^{\mathrm{AF}}(\boldsymbol{\nu}, \dot{\boldsymbol{\nu}})\right), \quad \mathcal{L}^{\mathrm{MDP}}=\boldsymbol{\sigma}: \dot{\boldsymbol{\epsilon}}-\boldsymbol{\sigma}: \dot{\boldsymbol{\epsilon}}^{\mathrm{p}}-\chi: \dot{\boldsymbol{\xi}}+\Phi^{\mathrm{AF}}(\boldsymbol{\nu}, \dot{\boldsymbol{\nu}}) .
$$

Bearing in mind the deviatoric character of quantities $\dot{\boldsymbol{\epsilon}}$ and $\dot{\boldsymbol{\xi}}$, the problem can also be written as:

$$
\min _{\dot{\boldsymbol{\nu}}}\left(\mathcal{L}^{\mathrm{MDP}}=\dot{\Psi}^{\mathrm{AF}}+\Phi^{\mathrm{AF}}(\boldsymbol{\nu}, \dot{\boldsymbol{\nu}})\right), \quad \mathcal{L}^{\mathrm{MDP}}=\boldsymbol{\sigma}: \dot{\boldsymbol{\epsilon}}-\overline{\boldsymbol{\sigma}}: \dot{\boldsymbol{\epsilon}}^{\mathrm{p}}-\overline{\boldsymbol{\chi}}: \dot{\boldsymbol{\xi}}+\Phi^{\mathrm{AF}}(\boldsymbol{\nu}, \dot{\boldsymbol{\nu}}) .
$$

Equation (35) is the counterpart of the MDP-problem (20) in terms of velocities and provides expressions for driving forces according to relationship $\boldsymbol{q}=\frac{\partial \Phi^{\mathrm{AF}}}{\partial \dot{\nu}}:$ 


$$
\begin{gathered}
\boldsymbol{q}_{\boldsymbol{\epsilon}^{\mathrm{p}}}=\overline{\boldsymbol{\sigma}}=\frac{\partial \Phi^{\mathrm{AF}}}{\partial \dot{\boldsymbol{\epsilon}}^{\mathrm{p}}}=\bar{\sigma}_{\mathrm{Y}} \frac{\dot{\boldsymbol{\epsilon}}^{\mathrm{p}}}{\left\|\dot{\boldsymbol{\epsilon}}^{\mathrm{p}}\right\|}+b \dot{\boldsymbol{\epsilon}}^{\mathrm{p}}+b \dot{\boldsymbol{\xi}}, \\
\boldsymbol{q}_{\boldsymbol{\xi}}=\bar{\chi}=\frac{\partial \Phi^{\mathrm{AF}}}{\partial \dot{\boldsymbol{\xi}}}=b \dot{\boldsymbol{\xi}}+b \dot{\boldsymbol{\epsilon}}^{\mathrm{p}} .
\end{gathered}
$$

A transformation of the system (36) and (37) yields the evolution equations identical to the ones from Sect. 4 (Eqs. 24$)$ and 250$)$ :

$$
\dot{\boldsymbol{\epsilon}}^{\mathrm{p}}=\lambda \frac{\overline{\boldsymbol{\sigma}}-\bar{\chi}}{\|\overline{\boldsymbol{\sigma}}-\bar{\chi}\|}, \quad \dot{\boldsymbol{\xi}}=-\dot{\boldsymbol{\epsilon}}^{\mathrm{p}}+\frac{1}{b} \bar{\chi} .
$$

Note that the insertion of (37) into (36) yields the intermediate result

$$
\overline{\boldsymbol{\sigma}}=\bar{\sigma}_{\mathrm{Y}} \frac{\dot{\boldsymbol{\epsilon}}^{\mathrm{p}}}{\left\|\dot{\boldsymbol{\epsilon}}^{\mathrm{p}}\right\|}+\chi \quad \Rightarrow \quad \overline{\boldsymbol{\sigma}}-\bar{\chi}=\bar{\sigma}_{\mathrm{Y}} \frac{\dot{\boldsymbol{\epsilon}}^{\mathrm{p}}}{\left\|\dot{\boldsymbol{\epsilon}}^{\mathrm{p}}\right\|},
$$

which is only valid for $\left\|\dot{\boldsymbol{\epsilon}}^{\mathrm{p}}\right\| \neq 0$. By taking the norm of $(39 \mathrm{~b}$, it follows that $\|\overline{\boldsymbol{\sigma}}-\overline{\boldsymbol{\chi}}\|=\bar{\sigma}_{\mathrm{Y}}$. This consequence indicates that dissipation potential (33) intrinsically includes the yield locus condition. Accordingly, it can be summed up that the dissipation potential (33) describes the same problem as the dissipation potential (21) along with the yield locus function (19).

Finally, the strong form corresponding to the Armstrong-Frederick problem can be recapitulated as follows:

$$
\begin{aligned}
& \text { Div } \boldsymbol{\sigma}=\mathbf{0}, \\
& \boldsymbol{\sigma}=\mathbb{C}:\left(\boldsymbol{\epsilon}-\boldsymbol{\epsilon}^{\mathrm{p}}\right), \\
& \dot{\boldsymbol{\epsilon}}^{\mathrm{p}}=\lambda \frac{\overline{\boldsymbol{\sigma}}-\bar{\chi}}{\|\overline{\boldsymbol{\sigma}}-\overline{\boldsymbol{\chi}}\|}, \\
& \dot{\boldsymbol{\chi}}=c\left(\dot{\boldsymbol{\epsilon}}^{\mathrm{p}}-\frac{1}{b} \overline{\bar{\chi}}\right), \\
& \Omega^{\mathrm{AF}}=\|\overline{\boldsymbol{\sigma}}-\overline{\boldsymbol{\chi}}\|-\bar{\sigma}_{\mathrm{Y}}, \\
& \lambda \geq 0, \Omega^{\mathrm{AF}} \leq 0, \lambda \Omega^{\mathrm{AF}}=0 .
\end{aligned}
$$

The previous system includes the equilibrium equation, the constitutive law, two evolution equations and the Karush-Kuhn-Tucker conditions defining the plastic domain. Equation (43) is derived by using the time derivative of definition (17) and rate $(38 \mathrm{~b}$ b. The body forces in the equilibrium equation are neglected. Evolution equation (42) and Karush-Kuhn-tucker conditions depend on plastic multiplier $\lambda$ which is typically determined from the consistency condition.

\section{Coupling of the Armstrong-Frederick model to the phase-field approach}

The coupling of the two methods starts by writing the Armstrong-Frederick energy in a form splitting the tension and compression part of elastic energy in order to introduce the damage influence as was done in Sect. 2 


$$
\Psi^{\mathrm{AF}}\left(\boldsymbol{\epsilon}^{\mathrm{e}}, \boldsymbol{\xi}\right)=\Psi^{\mathrm{el}}\left(\boldsymbol{\epsilon}^{\mathrm{e}}\right)+\Psi^{\mathrm{hard}}(\boldsymbol{\xi})=\Psi_{+}^{\mathrm{el}}\left(\boldsymbol{\epsilon}^{\mathrm{e}}\right)+\Psi_{-}^{\mathrm{el}}\left(\boldsymbol{\epsilon}^{\mathrm{e}}\right)+\Psi^{\mathrm{hard}}(\boldsymbol{\xi}) .
$$

However, damage also influences the hardening energy in the case where the tension mode is active. For this reason, the present model introduces a function distinguishing the pure compression mode from the pure tension and from the mixed modes

$$
\bar{\omega}=\left\{\begin{array}{lll}
\omega(d) & \text { if } & \max \left\{\left(\epsilon_{i}\right)_{i=1,2,3}, 0\right\}>0 \\
1 & \text { if } & \max \left\{\left(\epsilon_{i}\right)_{i=1,2,3}, 0\right\} \leq 0 .
\end{array}\right.
$$

By using this new notation, the coupled free energy is written as

$$
\Psi^{\mathrm{c}}\left(\epsilon^{\mathrm{e}}, d, \boldsymbol{\xi}\right)=\omega \Psi_{+}^{\mathrm{el}}\left(\boldsymbol{\epsilon}^{\mathrm{e}}\right)+\Psi_{-}^{\mathrm{el}}\left(\boldsymbol{\epsilon}^{\mathrm{e}}\right)+\bar{\omega} \Psi^{\mathrm{hard}}(\boldsymbol{\xi})
$$

and corresponding constitutive laws take the form

$$
\begin{gathered}
\boldsymbol{\sigma}=\frac{\partial \Psi^{\mathrm{c}}}{\partial \boldsymbol{\epsilon}}=\omega \frac{\partial \Psi_{+}^{\mathrm{el}}}{\partial \boldsymbol{\epsilon}}+\frac{\partial \Psi_{-}^{\mathrm{el}}}{\partial \boldsymbol{\epsilon}}=\omega \boldsymbol{\sigma}_{+}+\boldsymbol{\sigma}_{-} \\
\boldsymbol{\sigma}_{ \pm}=\frac{\partial \Psi_{ \pm}^{\mathrm{el}}}{\boldsymbol{\epsilon}}=\mathbb{C}: \boldsymbol{\epsilon}_{ \pm}^{\mathrm{e}}=\mathbb{C}:\left\langle\boldsymbol{\epsilon}-\boldsymbol{\epsilon}^{\mathrm{p}}\right\rangle_{ \pm} \\
\boldsymbol{\chi}=\frac{\partial \Psi^{\mathrm{c}}}{\partial \boldsymbol{\xi}}=-\bar{\omega} c \boldsymbol{\xi}
\end{gathered}
$$

where the spectral decomposition of elastic strains has the standard form

$$
\boldsymbol{\epsilon}_{ \pm}^{\mathrm{e}}=\left(\boldsymbol{\epsilon}-\boldsymbol{\epsilon}^{\mathrm{p}}\right)_{ \pm}=\sum_{i}\left\langle\epsilon_{i}^{\mathrm{e}}\right\rangle_{ \pm} \boldsymbol{n}_{i} \otimes \boldsymbol{n}_{i}=\sum_{i}\left\langle\left(\boldsymbol{\epsilon}-\boldsymbol{\epsilon}^{\mathrm{p}}\right)_{i}\right\rangle_{ \pm} \boldsymbol{n}_{i} \otimes \boldsymbol{n}_{i} .
$$

Bearing in mind stress definition 49 , the equilibrium equation turns into

$$
\operatorname{Div}\left[\omega(d) \boldsymbol{\sigma}_{+}+\boldsymbol{\sigma}_{-}\right]=\mathbf{0} .
$$

The damage influence on the dissipation functional related to the plastic deformations follows the same argumentation as in the case of the hardening energy, such that the coupled dissipation potential $\Phi^{\mathrm{c}}$ consists of two terms where the second term is weighted by the function $\bar{\omega}$

$$
\Phi^{\mathrm{c}}=\Phi^{\mathrm{d}}+\bar{\omega} \Phi^{\mathrm{AF}} .
$$

With the definitions (48) and (54), the minimization of the corresponding Lagrange function

$$
\mathcal{L}^{\mathrm{MDP}}=\dot{\Psi}^{\mathrm{c}}+\Phi^{\mathrm{c}}=\dot{\Psi}^{\mathrm{c}}+\Phi^{\mathrm{d}}+\bar{\omega} \Phi^{\mathrm{AF}}
$$

yields the following system of equations which, together with equilibrium equation (53), defines the strong form of the coupled problem 


$$
\begin{aligned}
& \boldsymbol{\sigma}=\omega(d) \boldsymbol{\sigma}_{+}+\boldsymbol{\sigma}_{-}, \\
& \dot{\boldsymbol{\epsilon}}^{\mathrm{p}}=\lambda \overline{\boldsymbol{N}}, \quad \overline{\boldsymbol{N}}=\frac{\overline{\boldsymbol{\sigma}}-\bar{\chi}}{\|\overline{\boldsymbol{\sigma}}-\bar{\chi}\|}, \\
& \dot{\boldsymbol{\xi}}=\frac{1}{b \bar{\omega}} \bar{\chi}-\dot{\boldsymbol{\epsilon}}^{\mathrm{p}}, \\
& \dot{\boldsymbol{\chi}}=-\dot{\bar{\omega}} c \boldsymbol{\xi}+c \bar{\omega}\left(\dot{\boldsymbol{\epsilon}}^{\mathrm{p}}-\frac{1}{b \bar{\omega}} \bar{\chi}\right), \\
& \Omega^{\mathrm{c}}=\|\overline{\boldsymbol{\sigma}}-\bar{\chi}\|-\bar{\omega} \bar{\sigma}_{\mathrm{Y}}, \\
& \lambda \geq 0, \Omega^{\mathrm{c}} \leq 0, \lambda \Omega^{\mathrm{c}}=0, \\
& \frac{g_{c}}{l}\left[d-l^{2} \Delta d\right]+\left[\omega^{\prime} \Psi_{+}^{\mathrm{el}}+\bar{\omega}^{\prime} \Psi^{\text {hard }}+k_{\mathrm{p}}\langle\dot{d}\rangle_{-}\right]=0 .
\end{aligned}
$$

Here, a shorter notation $\bar{N}=\frac{\overline{\boldsymbol{\sigma}}-\bar{\chi}}{\|\overline{\boldsymbol{\sigma}}-\overline{\boldsymbol{\chi}}\|}$ is introduced to denote the so-called direction tensor. Details on the derivation of evolution equations and of the yield criterion are provided in Appendix B.

\section{Determination of the plastic multiplier}

Evolution equation (57) and the Karush-Kuhn-Tucker conditions (61) depend on plastic multiplier $\lambda$ which is commonly determined from the consistency condition $\lambda \dot{\Omega}^{\mathrm{c}}=0$. An appropriate form of this condition is obtained by taking the time derivative of the yield locus formula

$$
\|\overline{\boldsymbol{\sigma}}-\bar{\chi}\|^{2}=\bar{\omega}^{2} \bar{\sigma}_{\mathrm{Y}}^{2} \quad \Rightarrow \quad(\overline{\boldsymbol{\sigma}}-\bar{\chi}):(\dot{\overline{\boldsymbol{\sigma}}}-\dot{\bar{\chi}})=0 .
$$

Since difference $(\overline{\boldsymbol{\sigma}}-\overline{\boldsymbol{\chi}})$ is deviatoric, the condition above also can be written as follows:

$$
(\overline{\boldsymbol{\sigma}}-\bar{\chi}):(\dot{\boldsymbol{\sigma}}-\dot{\chi})=0 .
$$

In a further step, the stress rate

$$
\dot{\boldsymbol{\sigma}}=\omega^{\prime} \boldsymbol{\sigma}_{+}+\omega(d) \mathbb{C}:\left(\dot{\boldsymbol{\epsilon}}-\dot{\boldsymbol{\epsilon}}^{\mathrm{p}}\right)_{+}+\mathbb{C}:\left(\dot{\boldsymbol{\epsilon}}-\dot{\boldsymbol{\epsilon}}^{\mathrm{p}}\right)_{-}
$$

along with Eqs. (57) and (59) is introduced in 64) which yields

$$
(\overline{\boldsymbol{\sigma}}-\bar{\chi}):\left[\omega^{\prime} \boldsymbol{\sigma}_{+}+\omega \mathbb{C}:[\dot{\boldsymbol{\epsilon}}-\lambda \overline{\boldsymbol{N}}]_{+}+\mathbb{C}:[\dot{\boldsymbol{\epsilon}}-\lambda \overline{\boldsymbol{N}}]_{-}+\dot{\bar{\omega}} c \boldsymbol{\xi}-c \bar{\omega} \lambda \overline{\boldsymbol{N}}+{ }_{b}^{c} \bar{\chi}\right]=0 .
$$

However, the split of the elastic strain rate into a positive and a negative part cannot be performed as a superposition of positive/negative parts of the total and plastic strains. For this reason, the previous equation can only be solved numerically by using an iterative procedure. For this purpose, the initial guess $\lambda_{0}$ is obtained by neglecting the terms including the stress rates

$$
(\overline{\boldsymbol{\sigma}}-\bar{\chi}):\left[\omega^{\prime} \boldsymbol{\sigma}_{+}+\dot{\bar{\omega}} c \boldsymbol{\xi}-c \bar{\omega} \lambda_{0} \overline{\boldsymbol{N}}+\frac{c}{b} \bar{\chi}\right]=0 .
$$

Thereafter, all subsequent steps follow the iteration rule for the calculation of the updated value $\lambda_{i}$ 


$$
(\overline{\boldsymbol{\sigma}}-\overline{\boldsymbol{\chi}}):\left[\omega^{\prime} \boldsymbol{\sigma}_{+}+\omega \mathbb{C}:\left[\dot{\boldsymbol{\epsilon}}-\lambda_{i-1} \overline{\boldsymbol{N}}\right]_{+}+\mathbb{C}:\left[\dot{\boldsymbol{\epsilon}}-\lambda_{i-1} \overline{\boldsymbol{N}}\right]_{-}+\dot{\bar{\omega}} c \boldsymbol{\xi}-c \bar{\omega} \lambda_{i} \overline{\boldsymbol{N}}+\frac{c}{b} \bar{\chi}\right]=0 .
$$

The iterative process stops if the prescribed accuracy is achieved.

\section{Numerical implementation}

\subsection{General approach}

The minimum principle of dissipation potential along with the time-incremental variational principles represents an important tool for the numerical solution of boundary value problems. Within this concept, the Lagrange function $\mathcal{L}^{\mathrm{MDP}}$ corresponding to the body $\Omega$ is integrated over a single time increment $\left[t_{n}, t_{n+1}\right]$. In its original form, this integral depends on external variables $(\boldsymbol{u})$, internal variables $(\boldsymbol{\nu})$ and on the rates of internal variables, so-called velocities $(\dot{\boldsymbol{\nu}})$. However, the velocities can be replaced by the Euler-forward approximation $\dot{\boldsymbol{\nu}}=\left(\boldsymbol{\nu}_{n+1}-\boldsymbol{\nu}_{n}\right) / \Delta t$, where $\Delta t=t_{n+1}-t_{n}$ is the time increment. As a consequence, the result only depends on discrete values of external and internal variables such that the time integration is performed as follows

$$
\int_{t_{n}}^{t_{n+1}} \int_{\Omega} \mathcal{L}^{\mathrm{MDP}} d V d t \approx \int_{\Omega}\left\{\Psi\left(\boldsymbol{\epsilon}_{n+1}^{\mathrm{e}}, \boldsymbol{\nu}_{n+1}\right)-\Psi\left(\boldsymbol{\epsilon}_{n}^{\mathrm{e}}, \boldsymbol{\nu}_{n}\right)+\Delta t \Phi\left(\boldsymbol{\nu}_{n+1},\left(\boldsymbol{\nu}_{n+1}-\boldsymbol{\nu}_{n+1}\right) / \Delta t\right)\right\} d V .
$$

Here, displacement $\boldsymbol{u}$ is the only external variable since an isothermal process is considered. In a further step, the minimization with respect to the displacements and internal variables in the current time step $n+1$ yields the sought solution. Within this procedure, term $\Psi\left(\boldsymbol{\epsilon}_{n}^{\mathrm{e}}, \boldsymbol{\nu}_{n}\right)$ can be neglected since it only depends on values in the previous time step $n$. By adding the potential of external forces $l\left(t_{n+1}, \boldsymbol{u}_{n+1}\right)$ in 69$)$, the new, combined Lagrangian is constructed

$\mathcal{L}^{\mathrm{comb}}=\int_{\Omega}\left\{\Psi\left(\boldsymbol{\epsilon}_{n+1}^{\mathrm{e}}, \boldsymbol{\nu}_{n+1}\right)+\Delta t \Phi\left(\boldsymbol{\nu}_{n+1},\left(\boldsymbol{\nu}_{n+1}-\boldsymbol{\nu}_{n+1}\right) / \Delta t\right)\right\} d V+l\left(t_{n+1}, \boldsymbol{u}_{n+1}\right)$.

Within the present work, the minimization of $(70)$ is performed by an approach consisting of two parts: the global level solution part and the material point solution part. The former calculates deformation and damage, whereas the latter evaluates the internal variables $\boldsymbol{\epsilon}_{\mathrm{p}}$ and $\boldsymbol{\xi}$ by using the predictor-corrector scheme. In the continuation, each solution part is explained separately. The indexes related to step $n+1$ are omitted in order to achieve a concise representation.

\subsection{Global solution part}

The definition of the global solution part (gl) starts with the reduced formulation of the Lagrangian by only including the reduced elastic energy and damage potential 
$\mathcal{L}^{\mathrm{gl}}=\int_{\Omega} \mathcal{L} d V=\int_{\Omega} \omega(d) \Psi_{+}^{\mathrm{el}}\left(\boldsymbol{\epsilon}^{\mathrm{e}}\right)+\Psi_{-}^{\mathrm{el}}\left(\boldsymbol{\epsilon}^{\mathrm{e}}\right) d V+\int_{\Omega} \Delta t \Phi^{\mathrm{d}}\left(d_{n}, \nabla d_{n}, d, \nabla d\right) d V+l(t, \boldsymbol{u})$

By using the expression for the crack surface density (1) and definition (5) the increment $\Delta \Phi^{\mathrm{d}}$ can be reconstructed as shown in Miehe et al. (2010a b)

$$
\Delta t \Phi^{\mathrm{d}}\left(d_{n}, \nabla d_{n}, d, \nabla d\right)=\frac{g_{c}}{2 l}\left(d^{2}-d_{n}^{2}\right)+\frac{g_{c} l}{2}\left(|\nabla d|^{2}-\left|\nabla d_{n}\right|^{2}\right)+\frac{k_{\mathrm{p}}}{2 \Delta t}\left\langle d-d_{n}\right\rangle_{-}^{2} .
$$

The FE-implementation now requires the derivatives of the Lagrangian $\mathcal{L}$. The first derivatives are required in order to form the residual

$$
\begin{gathered}
\partial_{\epsilon} \mathcal{L}=\omega \mathbb{C}: \epsilon_{+}^{\mathrm{e}}+\mathbb{C}: \epsilon_{-}^{\mathrm{e}}, \\
\partial_{d} \mathcal{L}=\omega^{\prime} \Psi_{+}^{\mathrm{el}}+\frac{g_{c}}{l} d+\frac{k_{\mathrm{p}}}{\Delta t}\left\langle d-d_{n}\right\rangle_{-}, \quad \partial_{\nabla d} \mathcal{L}=g_{c} l \nabla d,
\end{gathered}
$$

whereas the second derivatives are needed for the definition of the stiffness matrix

$$
\begin{array}{ll}
\partial_{\boldsymbol{\epsilon}}^{2} \mathcal{L}=\omega \mathbb{C}: \boldsymbol{I}_{+}^{\boldsymbol{\epsilon}_{+}^{\mathrm{e}}}+\mathbb{C}: \boldsymbol{I}_{-}^{\epsilon^{\mathrm{e}}}, & \partial_{\boldsymbol{\epsilon} d}^{2} \mathcal{L}=\omega^{\prime} \mathbb{C}: \boldsymbol{\epsilon}_{+}^{\mathrm{e}}, \\
\partial_{d d}^{2} \mathcal{L}=\omega^{\prime \prime} \Psi_{+}^{\mathrm{el}}+\frac{g_{c}}{l}+\frac{k_{\mathrm{p}}}{\Delta t} I^{d}, & \partial_{\nabla d \nabla d}^{2} \mathcal{L}=g_{c} l .
\end{array}
$$

These relationships use the following indicator functions

$$
\begin{gathered}
\boldsymbol{I}_{+}^{\epsilon^{\mathrm{e}}=} \frac{\partial \boldsymbol{\epsilon}_{+}^{\mathrm{e}}}{\partial \boldsymbol{\epsilon}^{\mathrm{e}}}=\sum_{i} I_{i+}^{\mathrm{e}} \boldsymbol{m}_{i} \otimes \boldsymbol{m}_{i}, \quad \boldsymbol{m}_{i}=\boldsymbol{n}_{i} \otimes \boldsymbol{n}_{i}, \\
\boldsymbol{I}_{-}^{\boldsymbol{\epsilon}^{\mathrm{e}}}=\frac{\partial \boldsymbol{\epsilon}_{-}^{\mathrm{e}}}{\partial \boldsymbol{\epsilon}^{\mathrm{e}}}=\sum_{i}\left(1-I_{i+}^{\mathrm{e}}\right) \boldsymbol{m}_{i} \otimes \boldsymbol{m}_{i}, \\
I_{i+}^{\mathrm{e}}=\left\{\begin{array}{lll}
1 & \text { if } & \epsilon_{i}>0, \\
0 & \text { if } & \epsilon_{i} \leq 0,
\end{array} \quad I^{d}=\left\{\begin{array}{lll}
1 & \text { if } & d<d_{n}, \\
0 & \text { if } & d \geq d_{n} .
\end{array}\right.\right.
\end{gathered}
$$

The remaining part of the numerical procedure at this level encompasses the standard steps typical of an FE-model. To this end, the bilinear shape functions corresponding to a quadrilateral element and staggered solution scheme are applied in the present work. This approach staggers between the displacement and the phase-field and is advantageous compared to the monolithic solution type due to its higher robustness. However, both approaches are well established nowadays as shown in works applying monolithic schemes (Miehe et al. 2010b Kuhn and Müller, 2010: Schlueter et al., 2014, Msekh et al., 2015) or using its counterpart (Miehe et al., 2010a| Borden et al., 2012 | Hofacker and Miehe, 2013). The standard Newton-Raphson procedure is applied for the solution of nonlinear systems of equations.

\subsection{Material point solution part}

The material point solution part evaluates internal variables $\epsilon^{\mathrm{p}}$ and $\boldsymbol{\xi}$ by using a predictor-corrector scheme if damage and deformations $(d, \boldsymbol{u})$ as well as rate 
$\dot{\bar{\omega}}=\omega^{\prime} \frac{\left(d-d_{n}\right)}{\Delta t}$ are known from the global level solution. The predictor step calculates the trial stress by assuming that plastic deformations do not change in comparison with the previous time step $n$

$$
\sigma^{\mathrm{tr}}=\omega(d) \mathbb{C}:\left(\boldsymbol{\epsilon}-\boldsymbol{\epsilon}_{n}^{\mathrm{p}}\right)_{+}+\mathbb{C}:\left(\boldsymbol{\epsilon}-\boldsymbol{\epsilon}_{n}^{\mathrm{p}}\right)_{-} .
$$

The trial stress is furthermore introduced in the yield locus function to check whether the prediction is true

$$
\Phi^{\mathrm{c}, \mathrm{tr}}=\left\|\overline{\boldsymbol{\sigma}}^{\mathrm{tr}}-\bar{\chi}_{n}\right\|-\bar{\omega} \bar{\sigma}_{\mathrm{Y}} .
$$

If the control value $\Phi^{\mathrm{c}, \mathrm{tr}}$ is negative, the prediction $(80)$ is correct and the plastic deformations do not evolve in the present step. Otherwise, a corrector step is needed to update the plastic deformations. The corrector step firstly calculates the plastic multiplier according to the scheme shown in Sect. 7 The initial value $\lambda_{0}$ is determined by solving the simplified problem (67)

$$
\lambda_{0}=\frac{\left(\overline{\boldsymbol{\sigma}}^{\mathrm{tr}}-\overline{\boldsymbol{\chi}}_{n}\right):\left[\omega^{\prime} \overline{\boldsymbol{\sigma}}_{+}^{\mathrm{tr}}+\dot{\bar{\omega}} c \boldsymbol{\xi}_{n}+\frac{c}{b} \overline{\boldsymbol{\chi}}_{n}\right]}{c \bar{\omega}\left(\overline{\boldsymbol{\sigma}}^{\mathrm{tr}}-\overline{\boldsymbol{\chi}}_{n}\right): \overline{\boldsymbol{N}}^{\mathrm{tr}}},
$$

whereas the later iterations follow the rule

$$
\begin{gathered}
\lambda_{i}=\frac{\left(\overline{\boldsymbol{\sigma}}^{\mathrm{tr}}-\overline{\boldsymbol{\chi}}_{n}\right): \boldsymbol{R}}{c \bar{\omega}\left(\overline{\boldsymbol{\sigma}}^{\mathrm{tr}}-\overline{\boldsymbol{\chi}}_{n}\right): \overline{\boldsymbol{N}}^{\mathrm{tr}}}, \\
\boldsymbol{R}=\left[\omega^{\prime} \boldsymbol{\sigma}_{+}^{\mathrm{tr}}+\omega \mathbb{C}:\left[\frac{\left(\boldsymbol{\epsilon}-\boldsymbol{\epsilon}_{n}\right)}{\Delta t}-\lambda_{i-1} \overline{\boldsymbol{N}}^{\operatorname{tr}}\right]_{+}+\mathbb{C}:\left[\frac{\left(\boldsymbol{\epsilon}-\boldsymbol{\epsilon}_{n}\right)}{\Delta t}-\lambda_{i-1} \overline{\boldsymbol{N}}^{\operatorname{tr}}\right]_{-}+\dot{\bar{\omega}} c \boldsymbol{\xi}_{n}+\frac{c}{b} \overline{\boldsymbol{\chi}}_{n}\right] .
\end{gathered}
$$

The previous expressions use the approximation $\dot{\boldsymbol{\epsilon}}=\frac{\left(\boldsymbol{\epsilon}-\boldsymbol{\epsilon}_{n}\right)}{\Delta t}$ and notation $\overline{\boldsymbol{N}}^{\mathrm{tr}}=$ $\frac{\left(\overline{\boldsymbol{\sigma}}^{\mathrm{tr}}-\overline{\boldsymbol{\chi}}^{\mathrm{n}}\right)}{\left\|\overline{\boldsymbol{\sigma}}^{\mathrm{tr}}-\overline{\boldsymbol{\chi}}^{\mathrm{n}}\right\|}$. Note that index $i$ is related to the iterative solution for $\lambda_{i}$, whereas index $n$ denotes the time step. Quantities without any index are related to the step $n+1$ or are constant. Finally, the solution of the iterative procedure (83)-(84) is denoted $\lambda=\lambda_{i}$ and used to update plastic deformations $\epsilon^{\mathrm{p}}$, driving forces $\chi$ and stress response $\sigma$

$$
\begin{array}{cc}
\dot{\boldsymbol{\epsilon}}_{\mathrm{p}}=\frac{\boldsymbol{\epsilon}^{\mathrm{p}}-\boldsymbol{\epsilon}_{n}^{\mathrm{p}}}{\Delta \mathrm{t}}=\lambda \overline{\boldsymbol{N}}^{\mathrm{tr}} & \Rightarrow \quad \boldsymbol{\epsilon}^{\mathrm{p}}=\Delta t \lambda \overline{\boldsymbol{N}}^{\mathrm{tr}}+\boldsymbol{\epsilon}_{n}^{\mathrm{p}}, \\
\dot{\boldsymbol{\xi}}=\frac{\boldsymbol{\xi}-\boldsymbol{\xi}_{n}}{\Delta t}=\frac{1}{b \bar{\omega}} \bar{\chi}_{n}-\dot{\boldsymbol{\epsilon}}_{\mathrm{p}} & \Rightarrow \quad \boldsymbol{\xi}=\frac{\Delta t}{b \bar{\omega}} \bar{\chi}_{n}-\Delta t \lambda \overline{\boldsymbol{N}}^{\mathrm{tr}}+\boldsymbol{\xi}_{n}, \\
\dot{\chi}=\dot{\bar{\chi}}=\frac{\overline{\boldsymbol{\chi}}-\overline{\boldsymbol{\chi}}_{n}}{\Delta \mathrm{t}}=-\dot{\bar{\omega}} c \boldsymbol{\xi}_{n}+c \bar{\omega} \dot{\boldsymbol{\epsilon}}_{\mathrm{p}}-\frac{c}{b} \chi_{n} & \Rightarrow \quad \chi=\bar{\chi}=-\Delta t \dot{\bar{\omega}} c \boldsymbol{\xi}_{n}+c \bar{\omega} \Delta t \lambda \overline{\boldsymbol{N}}^{\mathrm{tr}}-\frac{c \Delta t}{b} \bar{\chi}_{n}+\overline{\boldsymbol{\chi}}_{n}, \\
\boldsymbol{\sigma}=\omega(d) \mathbb{C}:\left(\boldsymbol{\epsilon}-\boldsymbol{\epsilon}^{\mathrm{p}}\right)_{+}+\mathbb{C}:\left(\boldsymbol{\epsilon}-\boldsymbol{\epsilon}^{\mathrm{p}}\right)_{-} .
\end{array}
$$

\section{Representative numerical examples}

The model developed inherits the advantages of both methods that it incorporates. That makes its application field large, as demonstrated in the subsequent sections 
Table 1: Elastic and plastic material parameters for CS and SS.

\begin{tabular}{lllll}
\hline Parameter & Value CS & Value SS & Unit & Name \\
\hline$E$ & 212910 & 197960 & {$[\mathrm{MPa}]$} & Young's modulus \\
$\nu$ & 0.3 & 0.3 & {$[-]$} & Poisson's ratio \\
$\sigma_{\mathrm{Y}}$ & 451 & 552 & {$[\mathrm{MPa}]$} & yield stress \\
$b$ & 5 & 6 & {$\left[\mathrm{Ns} / \mathrm{mm}^{2}\right]$} & pseudo-viscosity \\
$c$ & 30000 & 70000 & {$[\mathrm{~N} / \mathrm{mm}]$} & kinematic hardening parameter \\
\hline
\end{tabular}

studying the behavior of the cold-formed carbon steel (CS) and of the cold-formed stainless steel (SS). Among others, selected numerical examples deal with the purely elasto-plastic material behavior (Sect. 9.1), with the simulation of crack propagation on a notched sample (Sect. 9.2) and with the study of the life time in the LCF-mode (Sect 9.3).

\subsection{Elasto-plastic behavior of the carbon steel and of the stainless steel}

The first group of examples illustrating the application of the model studies a purely elasto-plastic material behavior under a cyclic load by neglecting the damage effects. To this end, simulations at a single material point are performed for parameter sets shown in Tab. 1. The prescribed strains gradually change from zero to $1 \%$ and thereafter decrease to $-1 \%$. The cycle again closes at zero load level. Two cycles with identical load paths are carried out. Each cycle takes $2 \mathrm{~s}$ to complete, whereas a single time step is $\Delta t=0.01 \mathrm{~s}$.

Simulations are carried out for two kinds of steel, namely for the cold-formed carbon steel (CS) and the cold-formed stainless steel (SS). The results are furthermore compared to the experimental findings by Nip et al. (2010). This comparison is presented in Fig. 1 and approves an excellent agreement. The stainless steel hardens stronger than the carbon steel, such that parameter $c$ is higher in this case. More details on the parameter identification in the context of the Armstrong-Frederick model are for example provided in Wolff et al. (2010); Lubarda and Benson (2002).

\subsection{Crack propagation on a notched sample}

The geometry of the sample for the second group of simulations is shown in Fig. 2 The chosen square plate has dimensions $1 \mathrm{~mm} \times 1 \mathrm{~mm}$ and a thickness of $1 \mathrm{~mm}$ and represents a cutout of a 3D body with the large thickness, which substantiates the assumption of plane strains (Fig. 2a). The model does not include any material length scale, such that sample size and prescribed displacements can be scaled in a straightforward manner. The left edge of the sample is fixed in horizontal direction. The vertical displacement at the bottom left corner is additionally suppressed. Horizontal displacements $\bar{u}_{x}$ are prescribed at the right edge. The plate has a vertical initial crack from the middle of the bottom edge to the midpoint of the sample. It is discretized by a mesh with approximately 22500 quadrilateral elements such that a fine discretization is performed in the areas where crack propagation is expected (Fig. $2 \mathrm{~b}$ ). In this area, the effective 

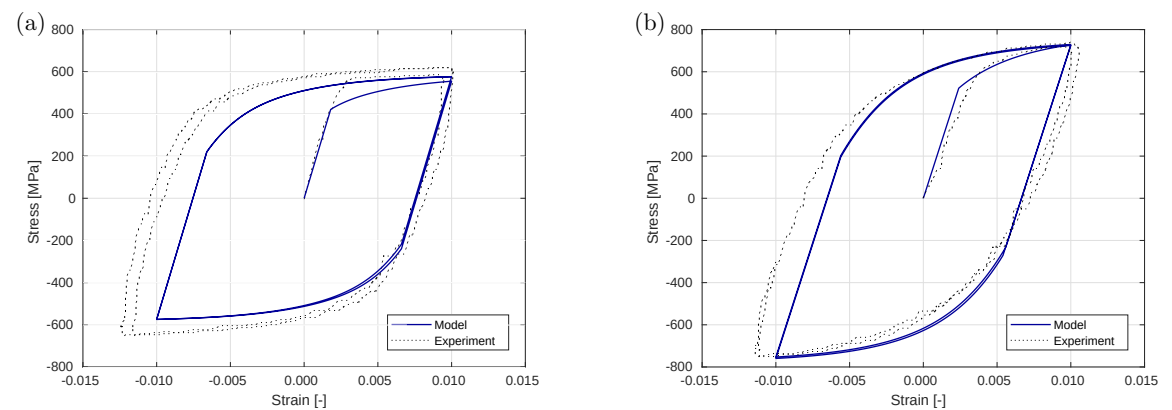

Figure 1: Comparison of experimental and numerical results at a material point. (a) Stress-strain hysteresis for carbon steel (CS). (b) Stress-strain hysteresis for stainless steel (SS). Plotted are the 11-components of the stress tensor and the strain tensor. Experimental results are taken from Nip et al. (2010).

element size $h$ fulfills the condition $h \approx 0.001 \mathrm{~mm}<l / 2$ and is significantly less than the minimum size required to achieve reasonable accuracy in the crack zone (Miehe et al., 2010b). Here, $l$ denotes the characteristic crack width typical of the phase field method.

(a)

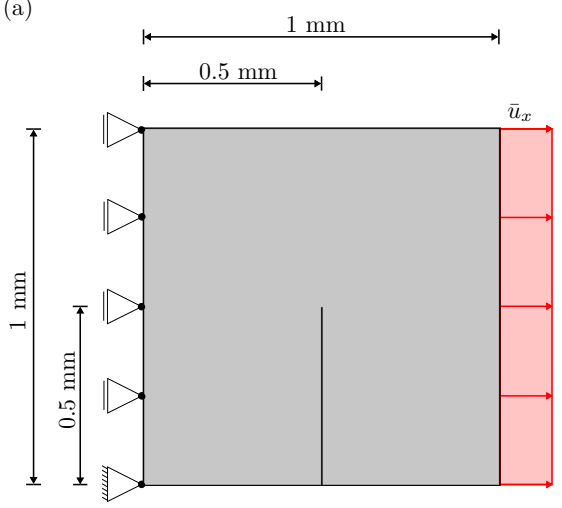

(b)



Figure 2: (a) Geometry of the notched plate with prescribed horizontal displacements $\bar{u}_{x}$. (b) Discretization of the plate with approximately 22500 elements.

The section simulates the crack propagation on the notched example and investigates the influence of material parameters on this process. The carbon steel is assumed as the representative material this time. Apart from elastic and plastic material parameters (Tab. 1), simulations require damage parameters. The values chosen to this end are listed in Tab. 2. Here, the values proposed by Miehe et al. (2010b) are used for the critical energy $g_{c}$ and characteristic crack width $l$. Constant $k_{\mathrm{p}}$ of the penalty function (Eq. (4) ) is chosen to be $1 \times 10^{8}$, which is sufficiently large to suppress the negative damage rate. Constant $k_{\mathrm{d}}$ has the value of $1 \times 10^{-8}$ and prevents the energy from becoming identical to zero in the case of full material damage. In all examples of this group, the load increment and damage parameters $g_{c}$ and $l$ are assumed such that the crack formation 
Table 2: Material parameters of the damage model.

\begin{tabular}{llll}
\hline Parameter & Value & Unit & Name \\
\hline$g_{c}$ & 0.27 & {$\left[\mathrm{~N} / \mathrm{mm}^{2}\right]$} & critical energy release rate \\
$l$ & 0.0375 & {$[\mathrm{~mm}]$} & characteristic crack width \\
$k_{\mathrm{p}}$ & $1 \times 10^{8}$ & {$[-]$} & penalty constant \\
$k_{\mathrm{d}}$ & $1 \times 10^{-8}$ & {$[-]$} & numerical constant \\
\hline
\end{tabular}

and propagation can be simulated within a fairly small number of time steps in order to reduce the computational effort and to provide a qualitative analysis of relevant phenomena according to the results from Miehe et al. (2010b).

The behavior of the notched sample (Fig. 2) is investigated for three load constellations: uniformly increasing tension, cyclic load with a tension and compression phase and cyclic load with an increasing amplitude in the tension regime.

In the first case, the prescribed displacement linearly increases up to the maximum value of $\bar{u}_{x}=0.005 \mathrm{~mm}$. The constant time increment is $\Delta t=0.005 \mathrm{~s}$ and the total loading time amounts to $1 \mathrm{~s}$. The results at a quadrature point of an element directly located at the end of the initial crack are monitored for the illustration in Fig. 3. This figure shows the change of damage and stress state during tension tests for different pairs of parameters $b$ and $c$. Here, rapidly hardening materials with a high hardening modulus $c$ lead to a faster increase of damage, whereas the higher pseudo-viscosity slows down the damage evolution and postpones softening. Damage increases from moderate values to the maximum, such that the material loses its strength and stress falls to the nearly zero value. The stress drop takes place in approximately $0.05 \mathrm{~s}$. Simulations are performed for different time increments in order to check the accuracy of the solution. For a time increment $\Delta t=0.005 \mathrm{~s}$ and less, identical results are achieved.
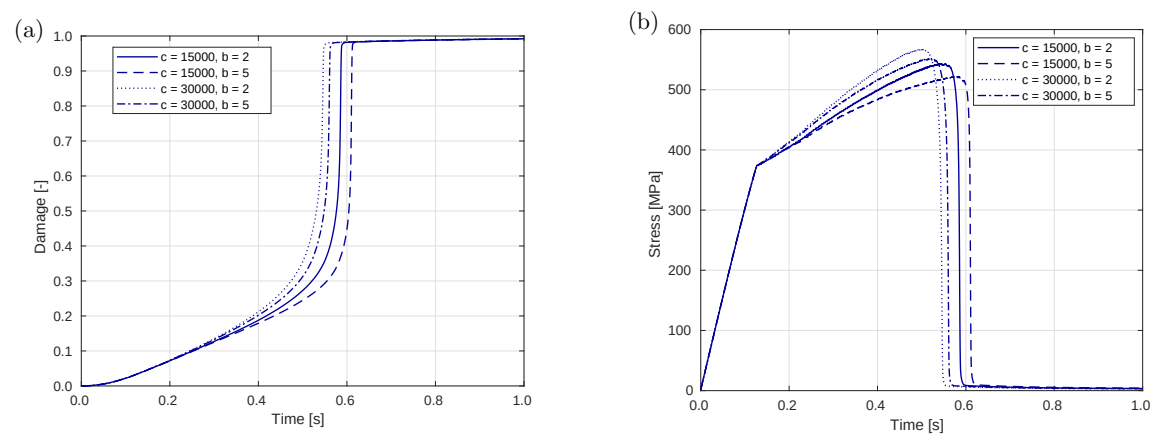

Figure 3: Results of tensile tests for different parameter pairs $b$ and $c$. Prescribed displacements uniformly change up to the value of $0.005 \mathrm{~mm}$. (a) Applied load as a function of time and damage evolution over time. (b) The 11-component of the stress tensor over the time. Hardening modulus $c$ is expressed in $\mathrm{N} / \mathrm{mm}$ and pseudo-viscosity $b$ is expressed in $\mathrm{Ns} / \mathrm{mm}^{2}$.

The same setup (Fig. 22 is used to investigate influences of a cyclic load as 
shown in Fig. 4. Here, two load cycles are performed with the total duration of $4 \mathrm{~s}$. The time increment is $\Delta t=0.005 \mathrm{~s}$. The horizontal displacement $\bar{u}_{x}$ changes in the range $[0.0025 \mathrm{~mm},-0.0025 \mathrm{~mm}]$, implying that a cycle includes both: the tension and the compression mode. The load amplitudes are constant in both cycles. Figure $4 a$ shows the load path and the damage evolution. The damage variable evolves during the tension phase, whereas the unloading and the compression mode do not affect it. This behavior goes back to the split of the energy into a tension and a compression part (Eq. (6p). Even though the same displacement is applied in every cycle, an increase in damage is observed during each loading cycle. Figure $4 \mathrm{~b}$ shows the strong influence of damage on the corresponding stress-strain hysteresis. Here, the Baushinger effect is hardly noticeable, although a displacement controlled test with constant amplitudes is simulated. Fig. $4 \mathrm{p}$ depicts the ratcheting rather than the Bauschinger effect.
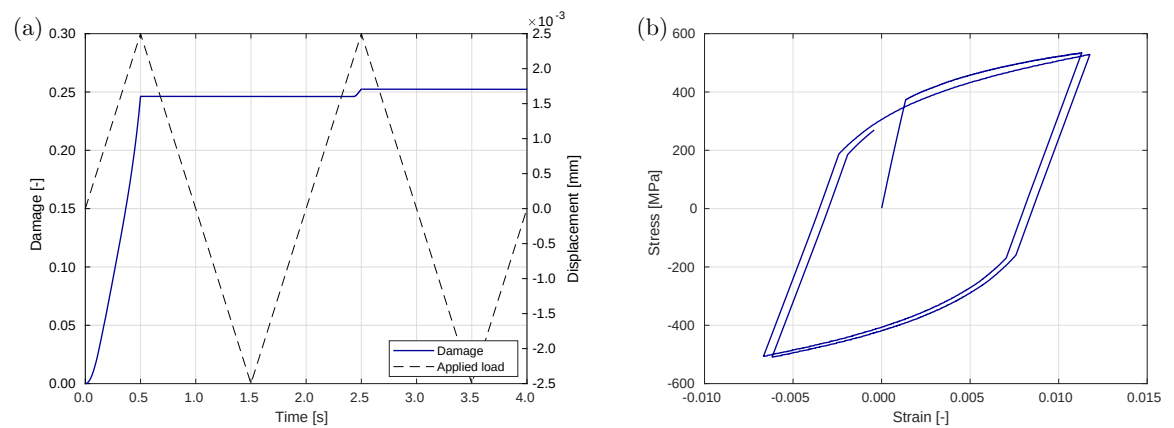

Figure 4: (a) Applied load as a function of time and the damage evolution for two load cycles. (b) The 11-component of the stress tensor over the 11-component of the strain tensor.

The last load constellation applied to the setup from Fig. 2 deals with the cyclic load in the tension regime with the increasing amplitude (Fig. 5). The amplitude increment amounts to $2 \times 10^{-4} \mathrm{~mm}$ per cycle. Time increment $\Delta t=0.005 \mathrm{~s}$ is assumed. Three damage contour plots are chosen presenting states of the crack during propagation for parameters $c=15000 \mathrm{~N} / \mathrm{mm}$ (Fig. 6a) and $c=30000 \mathrm{~N} / \mathrm{mm}$ (Fig. 6b). The first snapshot indicates the state at which damage variable $d$ reaches a value of one at the already existing crack tip. The second plot is taken when the crack has propagated roughly half the way through the plate, and the last plot is taken when the plate is fully torn in half. For the first parameter value $(c=15000 \mathrm{~N} / \mathrm{mm})$, the crack opening starts in the 19th load cycle and is fully propagated throughout the plate in the 23rd load cycle (Fig. 6a). For the second parameter value $(c=30000 \mathrm{~N} / \mathrm{mm})$, damage evolves earlier and faster: here, the complete crack propagation takes place within three load cycles (Fig. 6b). 


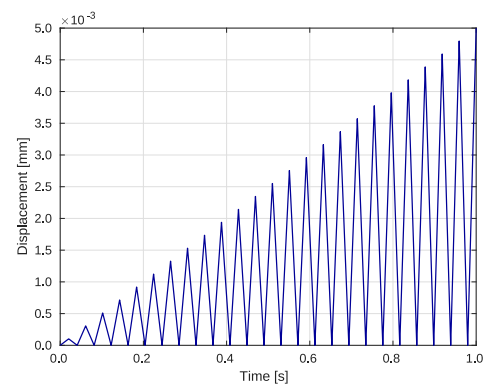

Figure 5: Increasing load amplitudes as a function of time.

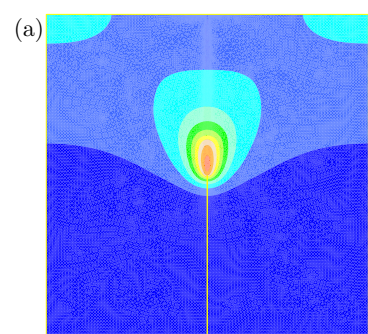

Cycle no. 19, time $0.755 \mathrm{~s}$

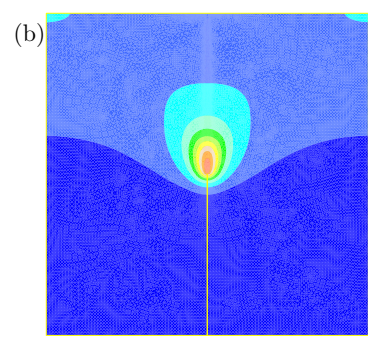

Cycle no. 17 , time $0.673 \mathrm{~s}$

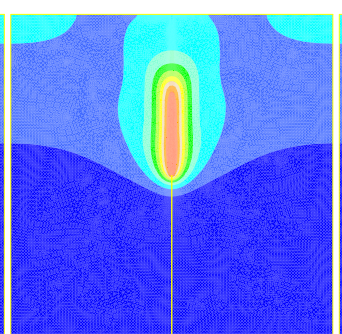

Cycle no. 21, time $0.837 \mathrm{~s}$

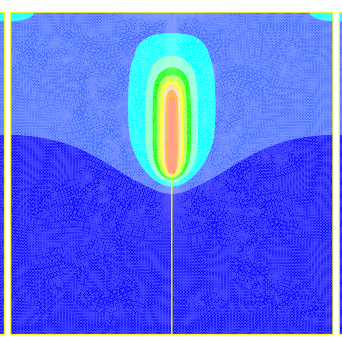

Cycle no. 18 , time $0.714 \mathrm{~s}$

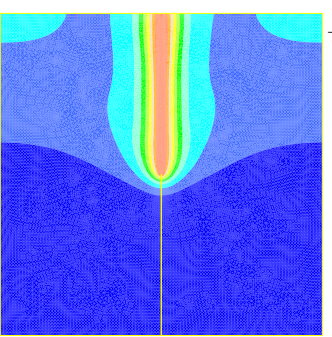

Cycle no. 23, time $0.918 \mathrm{~s}$

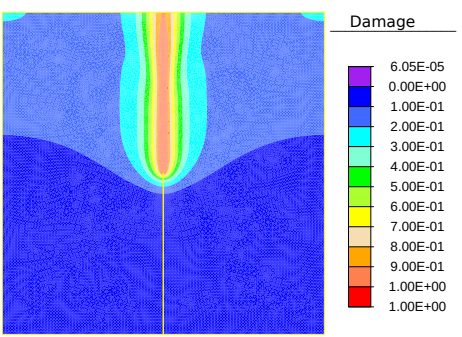

Cycle no. 19, time $0.755 \mathrm{~s}$

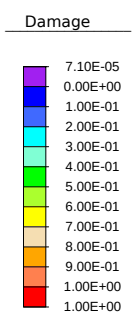

Figure 6: (a) Crack propagation takes place in five cycles $(c=15000 \mathrm{~N} / \mathrm{mm}$, $\left.b=2 \mathrm{Ns} / \mathrm{mm}^{2}\right)$. (b) Crack propagation takes place in three cycles $(c=30000$ $\left.\mathrm{N} / \mathrm{mm}, b=2 \mathrm{Ns} / \mathrm{mm}^{2}\right)$.

\subsection{Life time of the cold steel and of the stainless steel in the LCF-mode}

Amongst others, the model proposed enables the estimation of the life time in the LCF-mode. To this end, the number of cycles up to the total failure is evaluated dependent on the amplitude of the cyclic load applied. The latter is kept constant during the test. The material failure is caused by the material defects such as production process induced pores at a small length scale. In most cases, surface porosity is the critical factor for the fatigue phenomenon. However, the present paper assumes that different methods of the surface treatment can resolve this type of imperfection and that a defect in the bulk of material causes the crack initiation.

This part of the analysis assumes the sample geometry (Fig. 7a) proposed 
by Nip et al. (2010). The horizontal displacements are constrained at the left boundary and the cyclic horizontal displacements are prescribed at the right boundary. In addition, the vertical displacement at the lower left corner is constrained to suppress the rigid body motion. For the chosen boundary condition, the largest deformations are expected in the center of the sample, which is discretized by a fine mesh. The effective element size in this area is $\mathrm{h} \approx$ $0.25 \mathrm{~mm}$, such that the crack width length $l=0.6$ is chosen. The critical energy is set to $g_{c}=100 \mathrm{~N} / \mathrm{mm}^{2}$ which corresponds to a ductile material behavior, whereas the remaining material parameters are kept as in Sects. 9.1 and 9.2. Simulations are additionally performed for higher values of the hardening modulus $c$ in order to study the influence of this parameter. A defect is created in an element in the center of the specimen, which initiates crack growth in the otherwise homogeneous material. In this element, the values of the Young's modulus and the yield stress are set to $75 \%$ of the actual material parameters.

The very first step of the analysis applies a strain of $3 \%$. The assumed time increment is $\Delta t=4.28 \times 10^{-4} \mathrm{~s}$ and 2000 time steps simulate a single cycle. As expected, a vertical crack propagates through the middle of the sample, which is shown in Fig. 7p. Here, an early stage of the crack is already noticeable in the 47 th cycle, whereas the total failure occurs in cycle number 96 . Figure $7 \mathrm{~b}$ corresponds to the carbon steel with the hardening modulus $c=30000 \mathrm{~N} / \mathrm{mm}$.

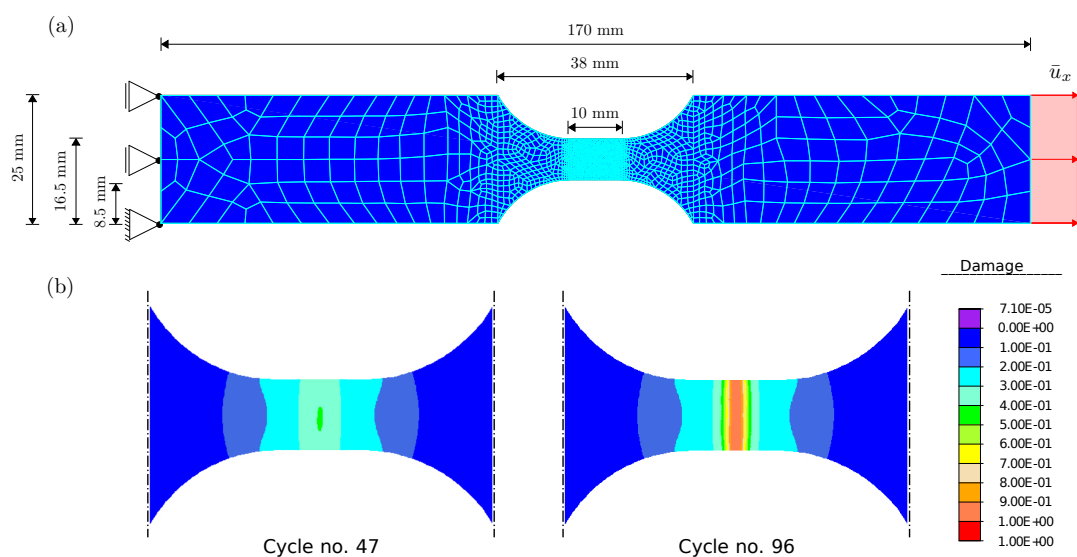

Figure 7: (a) Geometry of the simulated sample and prescribed boundary conditions. (b) Different stages of the crack propagation under the cyclic load. Simulations are performed for the carbon steel with the hardening modulus $c=30000 \mathrm{~N} / \mathrm{mm}$.

Furthermore, the tests are repeated by increasing the load amplitude to the strain of $4 \%, 5 \%, 6 \%$ and $7 \%$. The same setup (Fig. 7 a) is applied to this end. The number of time steps within a cycle is kept constant (2000), however, the time increment varies in the range from $\Delta t=4.28 \times 10^{-4} \mathrm{~s}$ to $\Delta t=1 \times 10^{-3} \mathrm{~s}$. Accordingly, the duration of one cycle takes the values between $0.856 \mathrm{~s}$ and $2 \mathrm{~s}$.

The results of simulations together with the experimental results by Nip et al. (2010) are presented in Fig. 8. First, the behavior of carbon steel is studied and compared to the Coffin-Manson curve relating the applied strains to the number of cycles, both in logarithmic scales (Fig. 8a). Numerical results show an excellent agreement with the experimental findings, in particular for 
higher strains. The dependence between the applied strains and the number of cycles is linear, however, the number of cycles is minimally overestimated. The discrepancy between the experimental and numerical results slightly increases with the decreasing strains. The same kind of simulations is repeated for a higher value of the hardening parameter, which leads to a significant decrease of the number of cycles up to the failure. This tendency goes back to the fact that a higher hardening modulus is related to the faster damage evolution and consequently causes a reduction of the number of cycles. Simulations for a higher hardening modulus approves the linear dependency between data, however, they indicate that a change of the strain amplitude has a higher influence on the change of number of cycles in this case.

The observations mentioned previously have also been approved by repeating the same kind of tests for the stainless steel as shown in Fig. 8b. Here, the Manson-Coffin curve has a slightly higher slope, but numerical results compared to the experimental ones show the same tendencies as for the carbon steel.
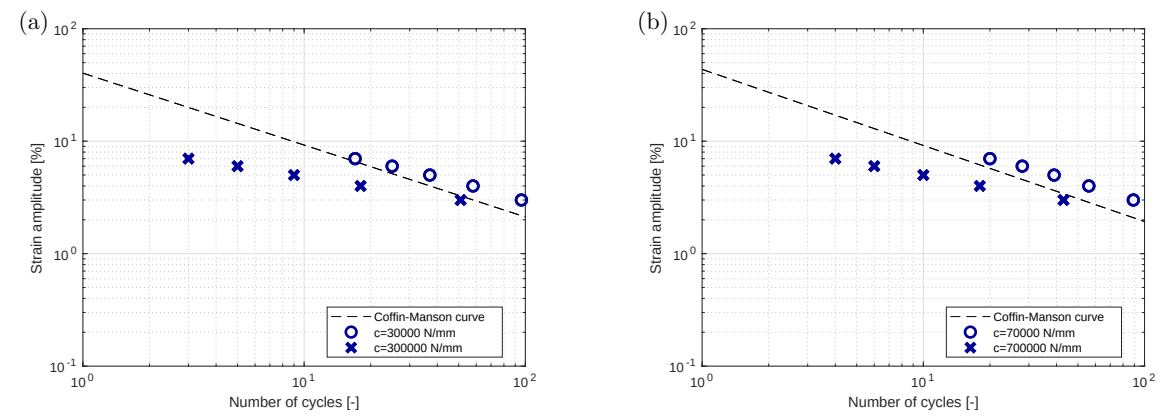

Figure 8: Comparison of the experimental Coffin-Manson curve Nip et al. 2010 ) to the numerical results. (a) Results for carbon steel with two different hardening parameters $c=30000 \mathrm{~N} / \mathrm{mm}$ and $c=300000 \mathrm{~N} / \mathrm{mm}$. (b) Results for the stainless steel with the hardening parameters $c=70000 \mathrm{~N} / \mathrm{mm}$ and $c=700000 \mathrm{~N} / \mathrm{mm}$.

\section{Conclusions and outlook}

The present work couples the phase field method of fracture to the ArmstrongFrederick model of plasticity with the kinematic hardening. The chosen concept inherits the advantages of both techniques and is aimed at the study of LCF effects in ductile materials. However, the numerical implementation of this promising approach faces several challenges, such as the definition of a unique framework for both setups, the derivation of coupled evolution equations, the distinction between tension and compression mode and the development of a computationally efficient algorithm. The basis for the phase field fracture model are the Griffith's theory and the dissipation potential relying on the assumption of a crack surface function. The derivation of evolution equations uses the minimum principle of the dissipation potential, which requires to express the dissipation potential of the classical Armstrong-Frederick model in terms of the internal variable rates by using the Legendre transformation. The model 
developed also takes into account that the unloading and the compression mode have no effect on the damage evolution. The approach is eventually implemented in the FE-program FEAP where the displacements and damage are calculated at the global level by using a staggered scheme, whereas inelastic internal variables are updated locally, at each Gauss point.

The application of the model is illustrated by three groups of examples related to the cold-formed carbon steel and the cold-formed stainless steel. The first group of tests investigates the purely elasto-plastic material behavior without the influence of damage and focuses on the numerical reconstruction of the stressstrain hysteresis due to a cyclic load. The second group of tests simulates the crack propagation on a notched sample and particularly analyses the influence of plastic parameters on the damage evolution. The simulations show that the rapidly hardening materials are prone to a faster damage evolution. The ability of the model to constrain the damage evolution in the unloading and compression stages of a loading cycle is demonstrated as well. The last group of tests studies the life time of the carbon steel in the LCF-mode and compares the numerical results to the experimental findings by Nip et al. (2010). The results show an excellent qualitative and quantitative agreement, and the linear dependency between the data in a logarithmic scale is approved.

The model proposed is a promising tool with regard to the simulation of fatigue effects, giving rise to many new issues. Among others, it can be extended to capture "hidden" aspects of the fatigue process such as the microcrack initiation based on the theory of persistent slip bands and the microcrack propagation along the crystallographic planes. Moreover, the additional effects typical of high and very high cycle fatigue, as well as the threshold for the damage initiation have to be incorporated. A comprehensive validation of the model with respect to the experimental results as well as its application and calibration for further materials are also envisaged.

\section{A Legendre transformation}

The formulation of the MDP in terms of rates of internal variables is based on Legendre transformation:

$$
\begin{gathered}
\Phi^{\mathrm{AF}}(\boldsymbol{\nu}, \dot{\boldsymbol{\nu}})=\max _{\boldsymbol{q}}\left\{\mathcal{L}^{\mathrm{LT}}=\boldsymbol{q}: \dot{\boldsymbol{\nu}}-\Phi^{\mathrm{AF}^{*}}(\boldsymbol{\nu}, \boldsymbol{q})\right\}, \\
\mathcal{L}^{\mathrm{LT}}=\boldsymbol{\sigma}: \dot{\boldsymbol{\epsilon}}^{\mathrm{p}}+\boldsymbol{\chi}: \dot{\boldsymbol{\xi}}-a\|\overline{\boldsymbol{\sigma}}-\bar{\chi}\|-\frac{1}{2 b}\|\overline{\boldsymbol{\chi}}\|^{2} .
\end{gathered}
$$

The corresponding stationary conditions have already been derived in Sect 5 (Eqs. 30 b and 31 b)

$$
\overline{\boldsymbol{\sigma}}=\bar{\sigma}_{\mathrm{Y}} \frac{\dot{\boldsymbol{\epsilon}}^{\mathrm{p}}}{\left\|\dot{\boldsymbol{\epsilon}}^{\mathrm{p}}\right\|}+b \dot{\boldsymbol{\xi}}+b \dot{\boldsymbol{\epsilon}}^{\mathrm{p}}, \quad \bar{\chi}=b \dot{\boldsymbol{\xi}}+b \dot{\boldsymbol{\epsilon}}^{\mathrm{p}}
$$

Their implementation in 90 now yields 


$$
\begin{gathered}
\mathcal{L}^{\mathrm{LT}}=\boldsymbol{\sigma}: \dot{\boldsymbol{\epsilon}}^{\mathrm{p}}+\boldsymbol{\chi}: \dot{\boldsymbol{\xi}}-a\|\overline{\boldsymbol{\sigma}}-\overline{\boldsymbol{\chi}}\|-\frac{1}{2 b}\|\overline{\boldsymbol{\chi}}\|^{2}= \\
=\bar{\sigma}_{\mathrm{Y}} \frac{\dot{\boldsymbol{\epsilon}}^{\mathrm{p}}}{\left\|\dot{\boldsymbol{\epsilon}}^{\mathrm{p}}\right\|}: \dot{\boldsymbol{\epsilon}}^{\mathrm{p}}+b \dot{\boldsymbol{\xi}}: \dot{\boldsymbol{\epsilon}}^{\mathrm{p}}+b \dot{\boldsymbol{\epsilon}}^{\mathrm{p}}: \dot{\boldsymbol{\epsilon}}^{\mathrm{p}}+b \dot{\boldsymbol{\xi}}: \dot{\boldsymbol{\xi}}+b \dot{\boldsymbol{\epsilon}}^{\mathrm{p}}: \dot{\boldsymbol{\xi}}-a\left\|\bar{\sigma}_{\mathrm{Y}} \frac{\dot{\boldsymbol{\epsilon}}^{\mathrm{p}}}{\left\|\dot{\boldsymbol{\epsilon}}^{\mathrm{p}}\right\|}\right\|-\frac{1}{2 b}\left[b^{2}\|\dot{\boldsymbol{\xi}}\|^{2}+2 b^{2} \dot{\boldsymbol{\xi}}: \dot{\boldsymbol{\epsilon}}^{\mathrm{p}}+b^{2}\left\|\dot{\boldsymbol{\epsilon}}^{\mathrm{p}}\right\|^{2}\right] \\
=\bar{\sigma}_{\mathrm{Y}}\left\|\dot{\boldsymbol{\epsilon}}^{\mathrm{p}}\right\|+b \dot{\boldsymbol{\xi}}: \dot{\boldsymbol{\epsilon}}^{\mathrm{p}}+b\left\|\dot{\boldsymbol{\epsilon}}^{\mathrm{p}}\right\|^{2}+b\|\dot{\boldsymbol{\xi}}\|^{2}+b \dot{\boldsymbol{\xi}}: \dot{\boldsymbol{\epsilon}}^{\mathrm{p}}-a \bar{\sigma}_{\mathrm{Y}}-\frac{b}{2}\|\dot{\boldsymbol{\xi}}\|^{2}-b \dot{\boldsymbol{\xi}}: \dot{\boldsymbol{\epsilon}}^{\mathrm{p}}-\frac{b}{2}\left\|\dot{\boldsymbol{\epsilon}}^{\mathrm{p}}\right\|^{2} \\
=\bar{\sigma}_{\mathrm{Y}}\left\|\dot{\boldsymbol{\epsilon}}^{\mathrm{p}}\right\|+\frac{b}{2}\left\|\dot{\boldsymbol{\epsilon}}^{\mathrm{p}}\right\|^{2}+\frac{b}{2}\|\dot{\boldsymbol{\xi}}\|^{2}+b \dot{\boldsymbol{\epsilon}}^{\mathrm{p}}: \dot{\boldsymbol{\xi}}-a \bar{\sigma}_{\mathrm{Y}}
\end{gathered}
$$

The last term is a constant and thus can be neglected, since the evolution equations are obtained by its minimization of the potential. Accordingly, the sought potential turns into

$$
\Phi^{\mathrm{AF}}(\boldsymbol{\nu}, \dot{\boldsymbol{\nu}})=\bar{\sigma}_{\mathrm{Y}}\left\|\dot{\boldsymbol{\epsilon}}^{\mathrm{p}}\right\|+\frac{b}{2}\left\|\dot{\boldsymbol{\epsilon}}^{\mathrm{p}}\right\|^{2}+\frac{b}{2}\|\dot{\boldsymbol{\xi}}\|^{2}+b \dot{\boldsymbol{\epsilon}}^{\mathrm{p}}: \dot{\boldsymbol{\xi}}
$$

\section{B Evolution equations of the coupled problem}

The potential of the coupled problem has the form

$$
\mathcal{L}^{\mathrm{MDP}}=\dot{\Psi}^{\mathrm{c}}+\Phi^{\mathrm{c}}=\dot{\Psi}^{\mathrm{c}}+\Phi^{\mathrm{d}}+\bar{\omega} \Phi^{\mathrm{AF}},
$$

where $\dot{\Psi}^{\mathrm{c}}$ is defined in Eq. (48), $\Phi^{\mathrm{d}}$ is defined by Eq. (5) and $\Phi^{\mathrm{AF}}$ by Eq. (33). Driving forces corresponding to $\epsilon^{\mathrm{p}}$ and $\boldsymbol{\xi}$ are then calculated as following derivatives

$$
\begin{aligned}
\boldsymbol{q}_{\boldsymbol{\epsilon}^{\mathrm{p}}}=\overline{\boldsymbol{\sigma}} & =\frac{\partial \Phi^{\mathrm{c}}}{\partial \dot{\boldsymbol{\epsilon}}^{\mathrm{p}}}=\bar{\omega}\left[\bar{\sigma}_{\mathrm{Y}} \frac{\dot{\boldsymbol{\epsilon}}^{\mathrm{p}}}{\left\|\dot{\boldsymbol{\epsilon}}^{\mathrm{p}}\right\|}+b \dot{\boldsymbol{\epsilon}}^{\mathrm{p}}+b \dot{\boldsymbol{\xi}}\right], \\
\boldsymbol{q}_{\boldsymbol{\xi}} & =\bar{\chi}=\frac{\partial \Phi^{\mathrm{c}}}{\partial \dot{\boldsymbol{\xi}}}=\bar{\omega}\left[b \dot{\boldsymbol{\xi}}+b \dot{\boldsymbol{\epsilon}}^{\mathrm{p}}\right],
\end{aligned}
$$

such that a transformation of the system 95 and 96 yields the evolution equations

$$
\dot{\boldsymbol{\epsilon}}^{\mathrm{p}}=\lambda \frac{\overline{\boldsymbol{\sigma}}-\bar{\chi}}{\|\overline{\boldsymbol{\sigma}}-\bar{\chi}\|}, \quad \dot{\boldsymbol{\xi}}=-\dot{\boldsymbol{\epsilon}}^{\mathrm{p}}+\frac{1}{\bar{\omega} b} \bar{\chi}
$$

whereas the insertion of 96 into 95 provides the yield locus equation

$\overline{\boldsymbol{\sigma}}=\bar{\omega} \bar{\sigma}_{\mathrm{Y}} \frac{\dot{\boldsymbol{\epsilon}}^{\mathrm{p}}}{\left\|\dot{\boldsymbol{\epsilon}}^{\mathrm{p}}\right\|}+\chi \quad \Rightarrow \quad \overline{\boldsymbol{\sigma}}-\bar{\chi}=\bar{\omega} \bar{\sigma}_{\mathrm{Y}} \frac{\dot{\boldsymbol{\epsilon}}^{\mathrm{p}}}{\left\|\dot{\boldsymbol{\epsilon}}^{\mathrm{p}}\right\|} \quad \Rightarrow \quad\|\overline{\boldsymbol{\sigma}}-\bar{\chi}\|=\bar{\omega} \bar{\sigma}_{\mathrm{Y}}$

Finally, constitutive law (51) is used to derive the evolution equation for the back stress:

$$
\dot{\chi}=-\dot{\bar{\omega}} c \boldsymbol{\xi}-\bar{\omega} c \dot{\boldsymbol{\xi}}=-\dot{\bar{\omega}} c \boldsymbol{\xi}+c \bar{\omega}\left(\dot{\epsilon}^{\mathrm{p}}-\frac{1}{b \bar{\omega}} \bar{\chi}\right)
$$




\section{Acknowledgments}

The authors are thankful to Frank Walther and Mustafa Awd (TU Dortmund University, Dortmund, Germany) for their helpful discussions on the fatigue behavior of metal materials.

\section{References}

Abdollahi, A., Arias, I., 2012. Phase-field modeling of crack propagation in piezoelectric and ferroelectric materials with different electromechanical crack conditions. J. Mech. Phys. Solids 60, 2100-2126.

Alessi, R., Marigo, J.J., Vidoli, S., 2014. Gradient damage models coupled with plasticity and nucleation of cohesive cracks. Arch. Rat. Mech. Anal. 214, 575-615.

Ambati, M., Gerasimov, T., De Lorenzis, L., 2015a. Phase-field modeling of ductile fracture. Comput. Mech. 55, 1017-1040.

Ambati, M., Kruse, R., De Lorenzis, L., 2015b. A phase-field model for ductile fracture at finite strains and its experimental verification. Comput. Mech. 57, 149-167.

Ambrosio, L., Tortorelli, V.M., 1990. Approximation of functionals depending on jumps by elliptic functionals via t-convergence. Commun- Pur. Appl. Math. 43, 999-1036.

Ambrosio, L., Tortorelli, V.M., 1992. On the approximation of free discontinuity problems. Boll. Un. Mat. Ital. B(7) 6, 105-123.

Armstrong, P.J., Frederick, C.O., 1966. A mathematical representation of the multiaxial Bauschinger effect. Central Electricity Generating Board and Berkeley Nuclear Laboratories 731.

Aygün, S., Klinge, S., 2020. Continuum mechanical modeling of strain-induced crystallization in polymers. Int. J. Solids Struct. 196-197, 129-139.

Bari, S., Hassan, T., 2001. Kinematic hardening rules in uncoupled modeling for multiaxial ratcheting simulation. Int. J. Plast. 17, 885-905.

Borden, M., Verhoosel, C., Scott, M., Hughes, T., Landis, C., 2012. A phase-field description of dynamic brittle fracture. Comput. Methods Appl. Mech. Eng. 217-220, 77-95.

Borden, M.J., Hughes, T.J.R., Landis, C.M., Anvari, A., Lee, I.J., 2016. A phasefield formulation for fracture in ductile materials: Finite deformation balance law derivation, plastic degradation, and stress triaxiality effects. Comput. Methods Appl. Mech. Eng. 312, 130-166.

Bourdin, B., Chambolle, A., 2000. Implementation of an adaptive finite-element approximation of the mumford-shah functional. Numer. Math. 85, 609-646. 
Bourdin, B., Chukwudozie, C., Yoshioka, K., 2012. A variational approach to the numerical simulation of hydraulic fracturing, in: SPE Annual Technical Conference and Exhibition, Society of Petroleum Engineers. pp. 1-9.

Bourdin, B., Francfort, G.A., Marigo, J.J., 2000. Numerical experiments in revisited brittle fracture. J. Mech. Phys. Solids 48, 797-826.

Bourdin, B., Marigo, J.J., Maurini, C., Sicsic, P., 2014. Morphogenesis and propagation of complex cracks induced by thermal shocks. Phys. Rev. Lett. $112,014301$.

Crismale, V., Lazzaroni, G., 2016. Viscous approximation of quasistatic evolutions for a coupled elastoplastic-damage model. Calc. Var. Partial Dif. 55, $1-54$.

Dettmer, W., Reese, S., 2004. On the theoretical and numerical modelling of Armstrong-Frederick kinematic hardening in the finite strain regime. Comput. Methods Appl. Mech. Eng. 193, 87-116.

Francfort, G.A., Marigo, J.J., 1998. Revisiting brittle fracture as an energy minimization problem. J. Mech. Phys. Solids. 46, 1319-1342.

Fraternali, F., 2007. Free discontinuity finite element models in two-dimensions for in-plane crack problems. Theor. Appl. Fract. Mec. 47, 274-282.

Freddi, F., Iurlano, F., 2017. Numerical insight of a variational smeared approach to cohesive fracture. J. Mech. Phys. Solids 98, 156-171.

Hofacker, M., Miehe, C., 2013. A phase field model of dynamic fracture: Robust field updates for the analysis of complex crack patterns. Int. J. Numer. Methods Eng. 93, 276-301.

Karma, A., Kessler, D.A., Levine, H., 2001. Phase-field model of mode III dynamic fracture. Phys. Rev. Lett. 87, 045501.

Khan, A.S., Jackson, K.M., 1999. On the evolution of isotropic and kinematic hardening with finite plastic deformation, Part I: Compression/tension loading of OFHC copper cylinders. Int. J. Plast. 15, 1265-1275.

Kobayashi, M., Ohno, N., 2002. Implementation of cyclic plasticity models based on a general form of kinematic hardening. Int. J. Numer. Meth. Eng. 53, $2217-2238$.

Kuhn, C., Müller, R., 2010. A continuum phase field model for fracture. Eng. Fract. Mech. 77, 3625-3634. Computational Mechanics in Fracture and Damage: A Special Issue in Honor of Prof. Gross.

Li, B., Peco, C., Millan, D., Arias, I., Arroyo, M., 2014. Phase-field modeling and simulation of fracture in brittle materials with strongly anisotropic surface energy. Int. J. Numer. Meth. Eng. 102, 711-727.

Lion, A., 2000. Constitutive modelling in finite thermoviscoplasticity: A physical approach based on nonlinear rheological models. Int. J. Plast. 16, 469-494. 
Lubarda, V., Benson, D., 2002. On the numerical algorithm for isotropic-kinematic hardening with the Armstrong-Frederick evolution of the back stress. Comput. Methods Appl. Mech. Eng. 191, 3583-3596.

Lührs, G., Hartmann, S., Haupt, P., 1997. On the numerical treatment of finite deformations in elastoviscoplasticity. Comput. Methods Appl. Mech. Eng. 144, $1-21$.

Maurini, C., Bourdin, B., Gauthier, G., V., L., 2013. Crack patterns obtained by unidirectional drying of a colloidal suspension in a capillary tube: Experiments and numerical simulations using a two-dimensional variational approach. Int. J. Fracture 184, 75-91.

Miehe, C., Aldakheel, F., Raina, A., 2016. Phase field modeling of ductile fracture at finite strains: A variational gradient-extended plasticity-damage theory. Int. J. Plast. 84, 1-32.

Miehe, C., Hofacker, M., Schänzel, L.M., Aldakheel, F., 2015. Phase field modeling of fracture in multi-physics problems. Part II. Coupled brittle-toductile failure criteria and crack propagation in thermo-elastic-plastic solids. Comput. Methods Appl. Mech. Eng. 294, 486-522.

Miehe, C., Hofacker, M., Welschinger, F., 2010a. A phase field model for rateindependent crack propagation: Robust algorithmic implementation based on operator splits. Comput. Methods Appl. Mech. Eng. 199, 2765-2778.

Miehe, C., Welschinger, F., Hofacker, M., 2010b. Thermodynamically consistent phase-field models of fracture: Variational principles and multi-field fe implementations. Int. J. Numer. Meth. Eng. 83, 1273-1311.

Mollica, F., Rajagopal, K.R., Srinivasa, A.R., 2001. The inelastic behavior of metals subject to loading reversal. Int. J. Plast. 17, 1119-1146.

Msekh, M., Sargado, J.M., Jamshidian, M., Areias, P., Rabczuk, T., 2015. Abaqus implementation of phase-field model for brittle fracture. Comput. Mater. Sci. 96, 472-484.

Narayan, S., Anand, L., 2019. A gradient damage theory of fracture of quasibrittle materials. J. Mech. Phys. Solids 129, 119-146.

Negri, M., Paolini, M., 2001. Numerical minimization of the mumford-shah functional. Calcolo 38, 67-84.

Nip, K.H., Gardner, L., Davies, C.M., Elghazouli, A.Y., 2010. Extremely low cycle fatigue tests on structural carbon steel and stainless steel. J. Constr. Steel Res. 66, 96-110.

Ohno, N., Wang, J.D., 1993. Kinematic hardening rules with critical state of dynamic recovery: Part I: Formulation and basic features for ratcheting behavior, Part II: Application to experiments of ratcheting behavior. Int. J. Plast. 9, 375-403.

Puzrin, A.M., Houlsby, G.T., 2001. Fundamentals of kinematic hardening hyperplasticity. Int. J. Solids Struct. 38, 3771-3794. 
Schlueter, A., Willenbücher, A., Kuhn, C., Müller, R., 2014. Phase field approximation of dynamic brittle fracture. Comput. Mech. 54, 1141-1161.

Schmidt, B., Fraternali, F., Ortiz, M., 2009. Eigenfracture: An eigendeformation approach to variational fracture. Multiscale Model. Sim. 7, 1237-1266.

Svendsen, B., Arndt, S., Klingbeil, D., Sievert, R., 1998. Hyperelastic models for elastoplasticity with nonlinear isotropic and kinematic hardening at large deformation. Int. J. Solids Struct. 35, 3363-3389.

Wheeler, M.F., Wick, T., Wollner, W., 2014. An augmented-lagrangian method for the phase-field approach for pressurized fractures. Comput. Methods Appl. Mech. Eng. 271, 69-85.

Wilson, Z.A., Borden, M.J., Landis, C.M., 2013. A phase-field model for fracture in piezoelectric ceramics. Int. J. Fracture 193, 135-153.

Wilson, Z.A., Landis, C.M., 2016. Phase-field modeling of hydraulic fracture. J. Mech. Phys. Solids 96, 264-290.

Wolff, M., Suhr, B., Simsir, C., 2010. Parameter identification for an Armstrong-Frederick hardening law for supercooled austenite of SAE 52100 steel. Comput. Mater. Sci. 50, 487-495. 